\title{
Petrography, geochemistry and depositional model of Ispikan Conglomerate, Makran Accretionary Prism, Southwest Pakistan
}

\author{
Muhammad Ahmed Farooqui ${ }^{1}$, Khalil-Ur-Rehman ${ }^{2}$, Amir Yaseen ${ }^{2}$, \\ Ghazala Roohi ${ }^{2}$, Muhammad Umar ${ }^{3, *}$ \\ ${ }^{1}$ COMSATS University Islamabad, Lahore Campus, Defence Road, \\ Off Raiwind Road, Lahore, Pakistan. \\ ${ }^{2}$ Earth Sciences Division, Pakistan Museum of Natural History, Pakistan Science Foundation, \\ Garden Avenue, Shakarparian, Islamabad, Pakistan. \\ ${ }^{3}$ Dept. of Earth Sciences the University of Haripur, Pakistan. \\ *Corresponding author: umarkhan09@yahoo.com
}

\begin{abstract}
Sedimentology, petrography, and geochemistry of the Ispikan Conglomerate of southwest Makran have been studied to establish its stratigraphic position, age, provenance, and depositional environment. Very thin to massive beds, poor sorting, no fabric, and poor grading are the common features. It is composed of mostly reworked, medium- to coarse-grained pebbly sandstone, siltstone, and discontinuous lenses of matrix- and clast-supported conglomerate. The sandstone is composed of angular to sub-angular, poorly sorted, immature grains having $\mathrm{Q}_{72} \mathrm{~F}_{13} \mathrm{~L}_{15}$ as average composition. Pebbles are of mostly metamorphic and acidic igneous origin. Large angular boulders $(\sim 1.5 \mathrm{~m})$ of sandstone indicate quick debris flow conditions. Geochemical discriminants suggest the derivation of Ispikan sediments from an active continental margin. The $\mathrm{Nb}$ and $\mathrm{Zr} / \mathrm{Th}$ and $\mathrm{Ba} / \mathrm{Y}$ values indicate the continental island arc setting, whereas a high $\mathrm{K} / \mathrm{Rb}$ ratio points to the derivation of detritus from an acid and intermediate source. Ispikan Conglomerate is interpreted as an Eocene olistostrome formed after a localized submarine debris flow triggered by slope failure.
\end{abstract}

Keywords: Conglomerate; geochemistry; Makran; olistostrome; provenance; sedimentology.

\section{Introduction}

The Ispikan Conglomerate is exposed as a small, isolated hill in southwestern Makran of Pakistan (Figure 1). Its age and stratigraphic position were hypothetically assigned by HSC (1960) as Paleocene based on cream-colored marl that resembled the Cretaceous Parh group of Indian continents. Geological Survey of Pakistan and other workers (Bender \& Raza, 1995; Malkani \& Mahmood, (2017) continued accepting this incorrect age and its wrong stratigraphic position. Kassi et al. (2007) renamed it as "Ispikan Group" and proposed the Late Cretaceous to Paleocene age based on field observations without commenting on its provenance and tectonic affiliation. We contest all previous interpretations that were heavily based on the presence of Parh-looking Cretaceous marl underneath Ispikan Conglomerate (Rehman, 2002; Farooqui \& Rehman, 2013). 
Makran coastal area and the vast unexplored Makran Accretionary Prism (MAP) have attained considerable importance due to increased economic and developmental activities under China-Pakistan Economic Corridor (C-PEC). These activities have triggered several geoscientific investigations focusing on the exploration and exploitation of oil, gas, minerals, water, and energy resources. Additionally, the stratigraphy has a direct bearing on the seismicity of the Makran coastal area (Penney et al., 2017; Smith et al., 2013). Hence re-examination of the stratigraphic position of lithologic units is important and necessary for successful and cost-effective exploration activities. The purpose of this article is to revisit and establish the age, stratigraphic position, origin, and depositional environment of Ispikan Conglomerate for a better understanding of the subsurface geology of the area.

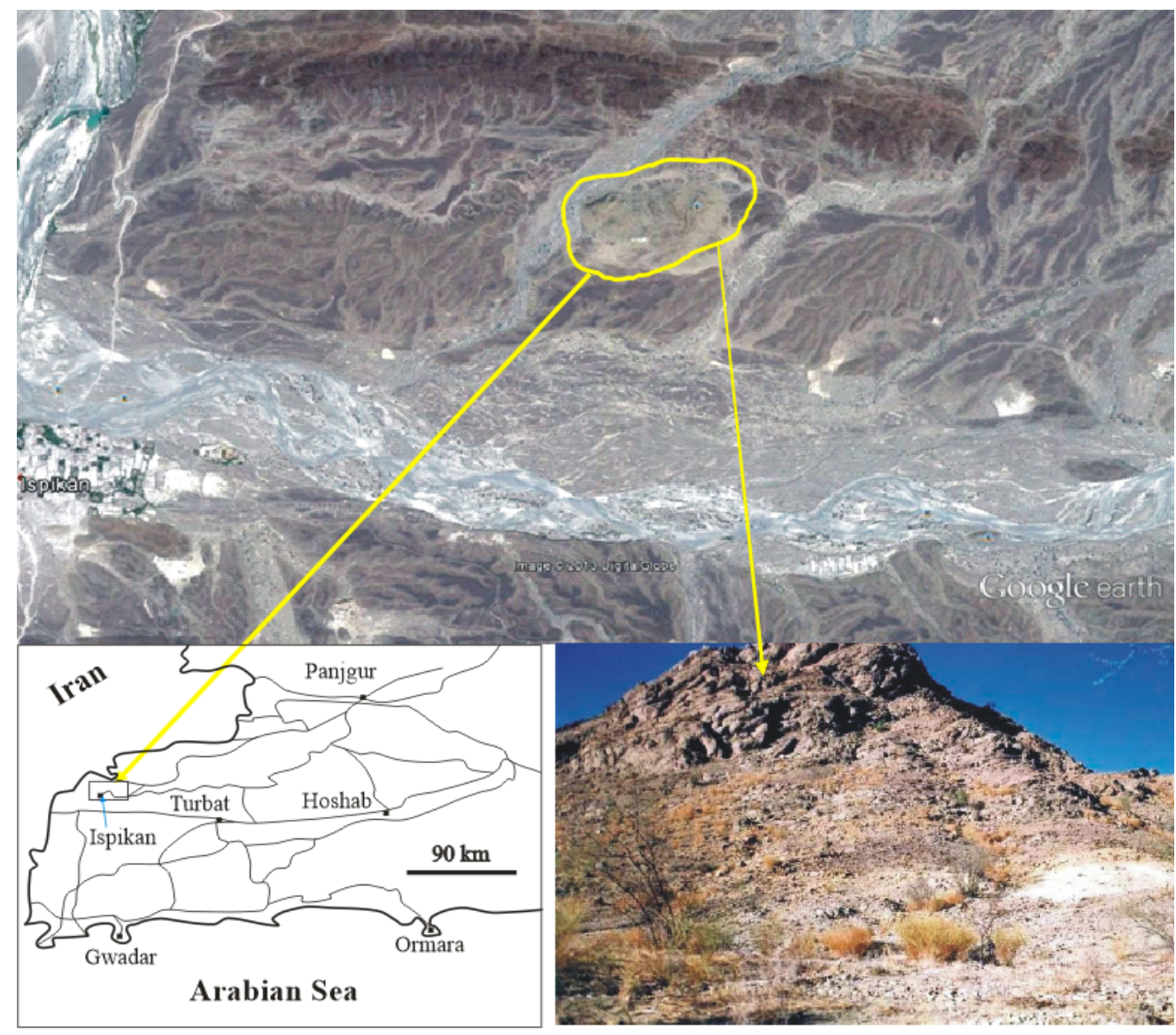

Fig.1. Satellite imagery and location map of the Ispikan outcrop. Also shown is the photograph of the southern side of the outcrop (looking towards north). 


\section{Regional Geology}

The Geology of Makran is dominated by subduction-related tectonics and sedimentation since the Cretaceous (McCall \& Kidd, 1982; Rehman, 2002; Burg, 2018; Shah et al., 2021). The Makran region is the southern part of a well-exposed large accretionary prism that is mostly filled with thick sequences of flysch, locally grading into orogenic molasses. In the west, the accretionary prism is truncated by the Oman Line from the oil-producing Zagros region of Iran, whereas in the north, it is bordered by the Chaghi-Raskoh Arc-a metallogenic province and the associated Mashkhel forearc basin (Farhoudi \& Karig, 1977; Raza \& Alam, 1983; Platt \& Leggett, 1986). The eastern boundary of the prism lies along the Chaman-Nushki and Ornach-Nal fault system which is an active western boundary of the north-moving Indo-Pak continental plate (Figure 2).

\section{Materials and Methods}

The single outcrop of the Ispikan Conglomerate was measured and sampled at two locations: eastern section at $26^{\mathrm{O}} 24^{/} 21.25^{/ /} \mathrm{N}, 62^{\mathrm{O}} 23^{/} 80.32^{/ /} \mathrm{E}$ and southern section at $26^{\mathrm{O}} 24^{/} 09.68^{/ /} \mathrm{N}, 62^{\mathrm{O}} 23^{\prime}$ $56.84^{/ /} \mathrm{E}$ that are $110 \mathrm{~m}$ and $135 \mathrm{~m}$ thick respectively. Description of the samples and measured sections are given in Appendix-1 and 2 respectively. Thin sections of the samples were studied and point counting was carried out under a petrographic microscope using the traditional GazziDickinson method (Dickinson, 1988). Additionally, 28 samples were analyzed for major oxides and trace element geochemistry. Analyses were carried out at Geosciences Laboratories, Geological Survey of Pakistan, Islamabad, on WD-XRF using glass beads and powder pellets.

\section{Results}

\subsection{Sedimentology}

Bedding attitude in the basal part of the eastern section varies between $22^{\circ}$ and $33^{\circ} \mathrm{NW}$, however, the beds in the upper part have a relatively gentler dip. In the southern section, bedding is almost flat having a dip angle between $10^{\circ}$ and $15^{\circ} \mathrm{NW}$. Beds are mostly composed of medium to light brownish gray, medium- to very coarse-grained pebbly sandstone (Figure 3a). Boulders and cobbles are repeatedly present at four positions. Similarly, large-scale crossbedding that appears to be hummocky, is also repeated four times. Stratification and gradation are poorly developed. Conglomerate beds are mostly disorganized, but parallel fabric and imbricated structures are also occasionally present (Figures 3b-c). Sandstone beds with crossbedding are common. Pebbly sandstone is the most dominant lithology where beds are separated by thin laminae of claystone and siltstone. Pebbles are spread randomly with vague organization and variable concentration. Rounded 
to well-rounded clasts are spherical, elliptical, discoidal, tabular, and elongate in shape. Limestone, granite, quartz, and quartzite pebbles are angular to very angular whereas andesite clasts are rounded to well-rounded. In some cases, pebbly sandstone is present in the form of lenses within a mediumto coarse-grained sandstone. Laminae of light gray to light yellowish-brown siltstone and claystone are commonly present between the two beds. The base of the exposed eastern section has scoured contact with the underlying cream-colored shale (Figure 3d). Detailed sedimentary features of Ispikan Conglomerate are indicated in Figure 4.

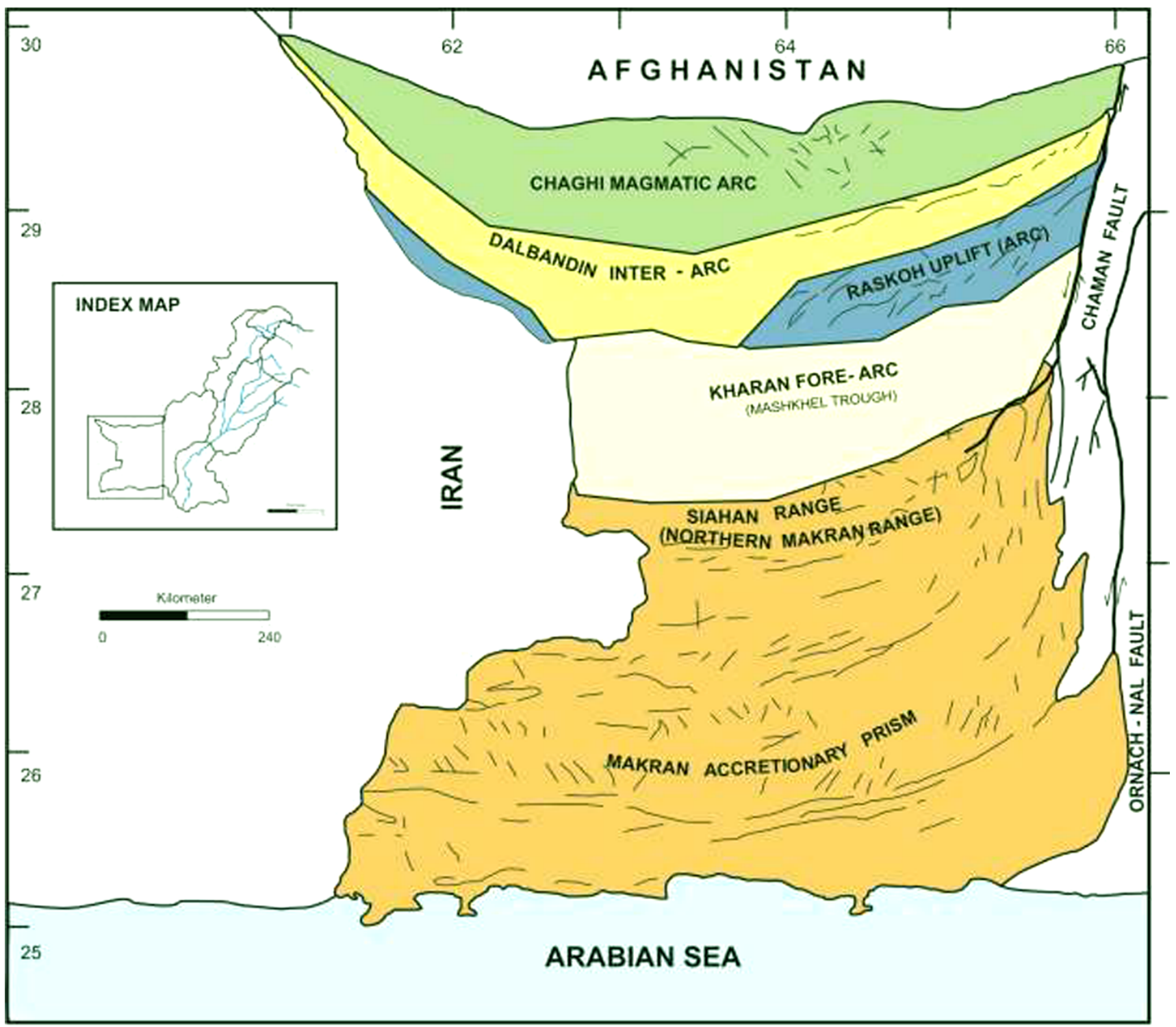

Fig.2. Generalized tectonic map (HSC, 1960) of Balochistan showing major tectonic segments of the Makran Accretionary Prism and Makran-Chaghi-Raskoh Arc Trench system. 


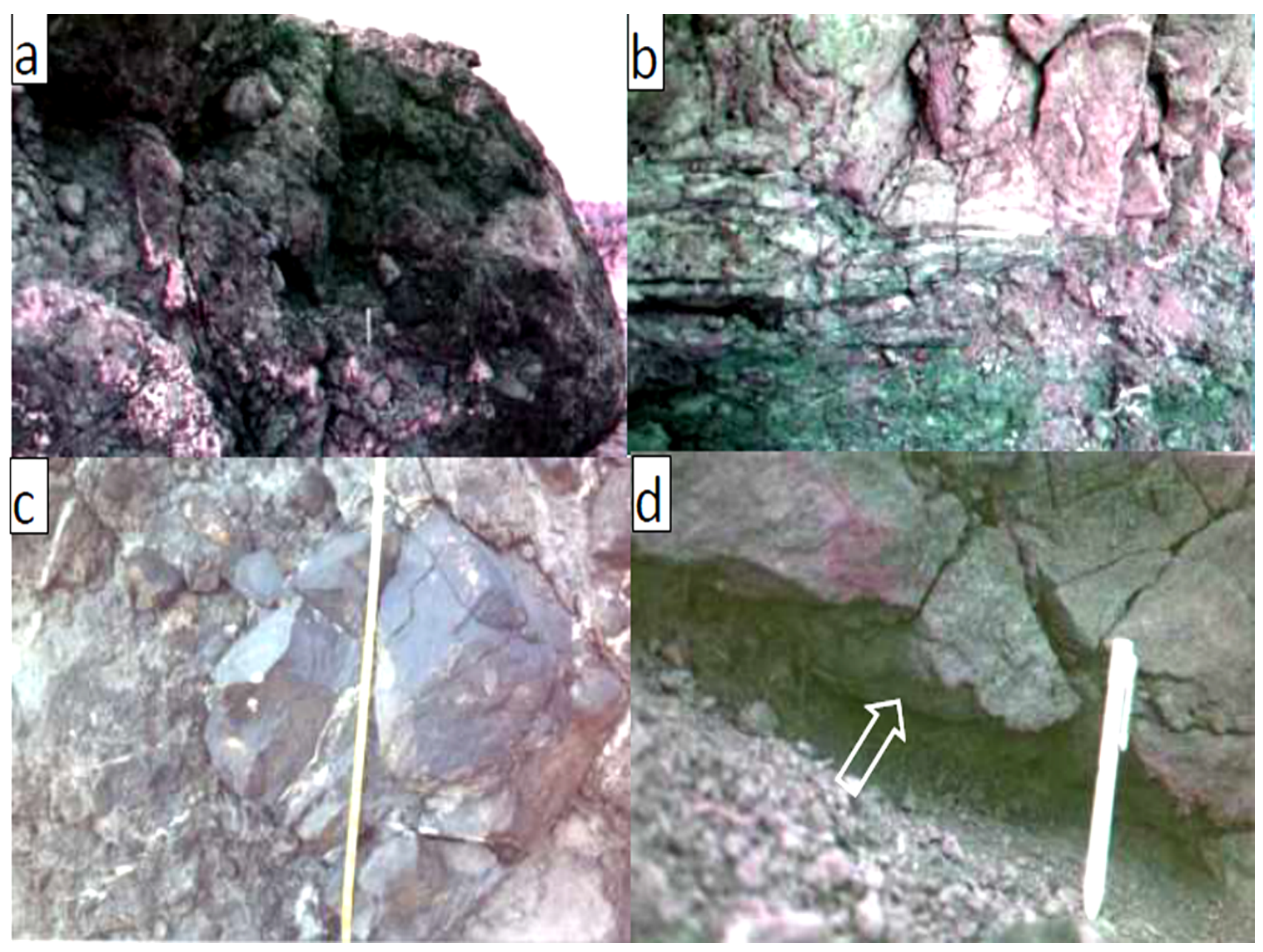

Fig. 3. (a): A typical conglomerate bed of Ispikan Conglomerate showing characters of olistrostrome. Note the angular and poorly sorted olistoliths. (b) structureless sandstone bed of the Ispikan Conglomerate (White pen in the middle is $12 \mathrm{~cm}$ long). (c) a large angular boulder (Olistolith) with in the Ispikan Conglomerate, (Scale: App.120 cm from base to top), (d) Scoured basal contact (arrow pointing toward the base) of Ispikan Conglomerate with Wakai Limestone. 


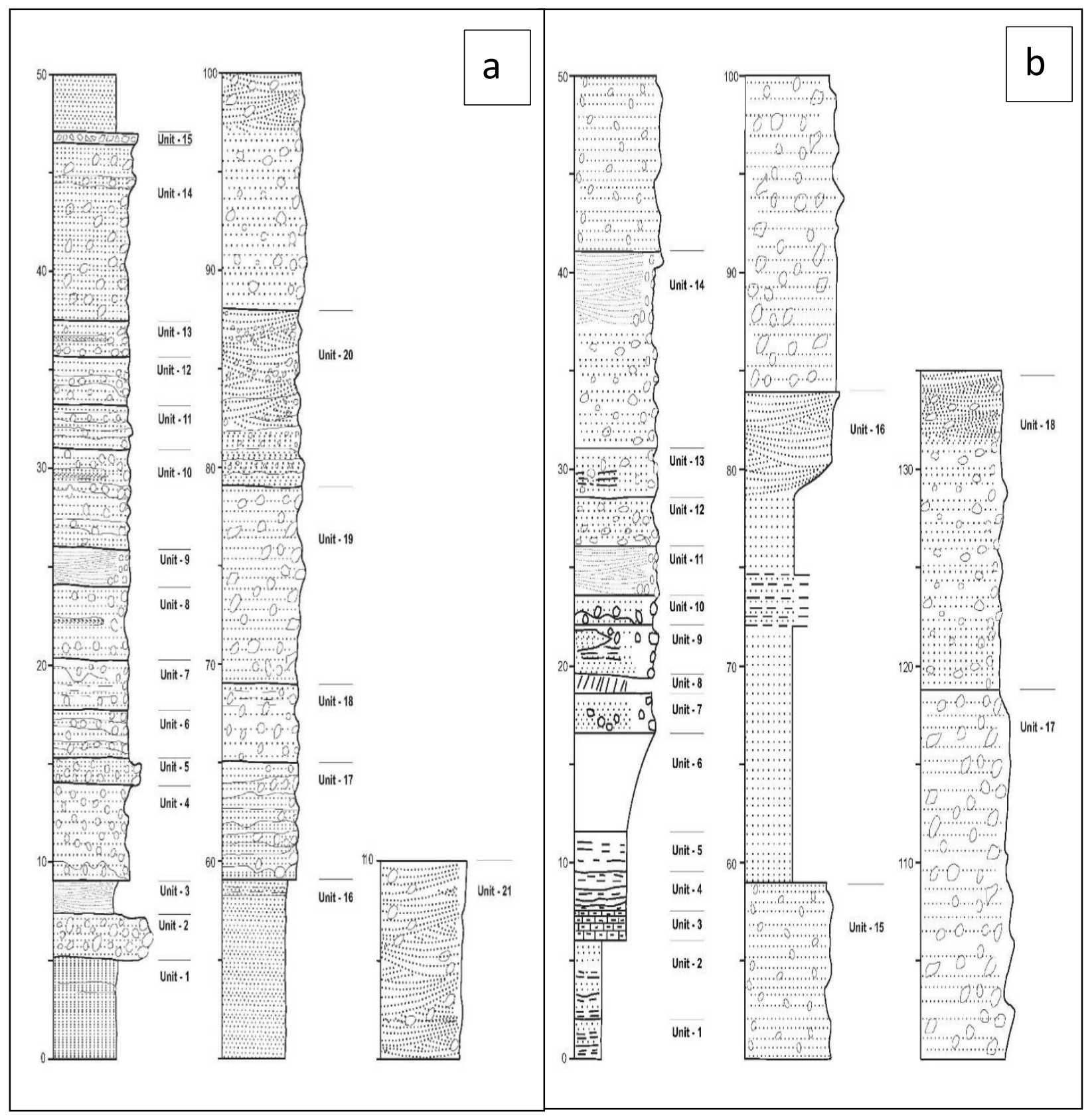

Fig. 4. Graphic logs of the litho-sedimentological characters of Ispikan Conglomerate. a) Eastern Section and b) Southern Section. Detailed descriptions of the sedimentological and lithological characters are given in Appendix-2.

\subsection{Petrography}

Out of fifty-two samples collected, only forty samples were found suitable for point counting. The samples are composed of moderately sorted, sub-angular to well-rounded dominantly mediumgrained sandstones having 53-85\% grains, 3-42\% matrix, and 4-14\% cement (Figures 5a-e). Framework grains are undulatory and non-undulatory monocrystalline quartz (Qmu and Qmnu), 
polycrystalline quartz (Qp), plagioclase (P), Potassium feldspar $(\mathrm{K})$ and rock fragments. Quartz is the most abundant framework grain constituting on average $68 \%$ of the rock volume. Fluid globules and tiny gas bubbles are present in some of the Qmnu suggesting their igneous origin. Polycrystalline quartz grains with straight intercrystallite boundaries are in smaller quantities.

Plagioclase grains are slightly too deeply altered (sericitized), angular to sub-rounded, medium- to fine-grained and make $7.4 \%$ of the total detrital grains. K-feldspar grains make $6.5 \%$ of the rock volume and are generally medium- to fine-grained, moderately sorted, fresher and less altered than plagioclase.

Rock fragments are present in medium- to coarse-grained samples. The sedimentary rock fragments (SRFs) are of two types: intrabasinal mud clasts (Figures 5a and 5c) and extrabasinal limestone fragments that are composed of sparry calcite and siltstone. Mud clasts are medium- to fine-grained and composed of claystone, silty claystone, and siltstone. The limestone fragments are medium- to coarse-grained, poorly sorted and are composed of sparry calcite and micrite (Figure $5 \mathrm{~b})$. The plutonic clasts are sub-angular to sub-rounded, moderately sorted and are composed of quartz and k-feldspar. Volcanic rock fragments (VRFs) are of two types, medium- to coarse-grained, andesite fragments containing plagioclase phenocrysts in volcanic glass and medium to finegrained, mostly altered volcanic glass. Metamorphic rock fragments are polycrystalline quartz grains with crenulated inter-crystallite boundaries (Figures $5 \mathrm{~d}-\mathrm{e}$ ).

\subsection{Geochemistry}

Geochemical analysis of siliciclastic rocks has become routine work in the study of clastic rocks. The results are mainly used for defining the tectonic setting of the sedimentary basin. Geochemistry also allows the tectonic setting of metasediments to be identified despite the loss of original petrographical detail (Haughton et al., 1991, Huang et al., 2018).

Twenty-eight samples were analyzed geochemically (Table 2). Using the $\mathrm{K}_{2} \mathrm{O}$ and $\mathrm{Na}_{2} \mathrm{O}$ parameters of Crook (1974), the samples are classified as quartz intermediate (Figure 6). The analyzed samples are characterized as low- $\mathrm{SiO}_{2}$, low- $\mathrm{TiO}_{2}$, low- $\mathrm{Na} 2 \mathrm{O}$, low- $\mathrm{P}_{2} \mathrm{O}_{5}$, medium- $\mathrm{K}_{2} \mathrm{O}$, high- $\mathrm{Fe}_{2} \mathrm{O}_{3}$, high- $\mathrm{CaO}$, high- $\mathrm{MgO}$, and very high-MnO. Large ion lithofile elements (LILE) such as $\mathrm{Rb}, \mathrm{Sr}$, and $\mathrm{Th}$ and other trace elements show a range of variable abundance. Closer observation revealed that based on $\mathrm{Ti}, \mathrm{Fe}, \mathrm{Mg}, \mathrm{Ca}$, and $\mathrm{K}$ oxides the samples can be divided into further groups. Ti abundance shows two distinct groups here designated as low-Ti and high-Ti sandstones. Most of the samples fall under the low-Ti category, whereas only two samples are of the high-Ti category. Similarly, based on $\mathrm{Fe}, \mathrm{Mg}, \mathrm{Ca}$, and $\mathrm{K}$ contents, the samples can be divided into low- and highelement categories. Other elements i.e., P, Al, Mn, Na are randomly distributed. 

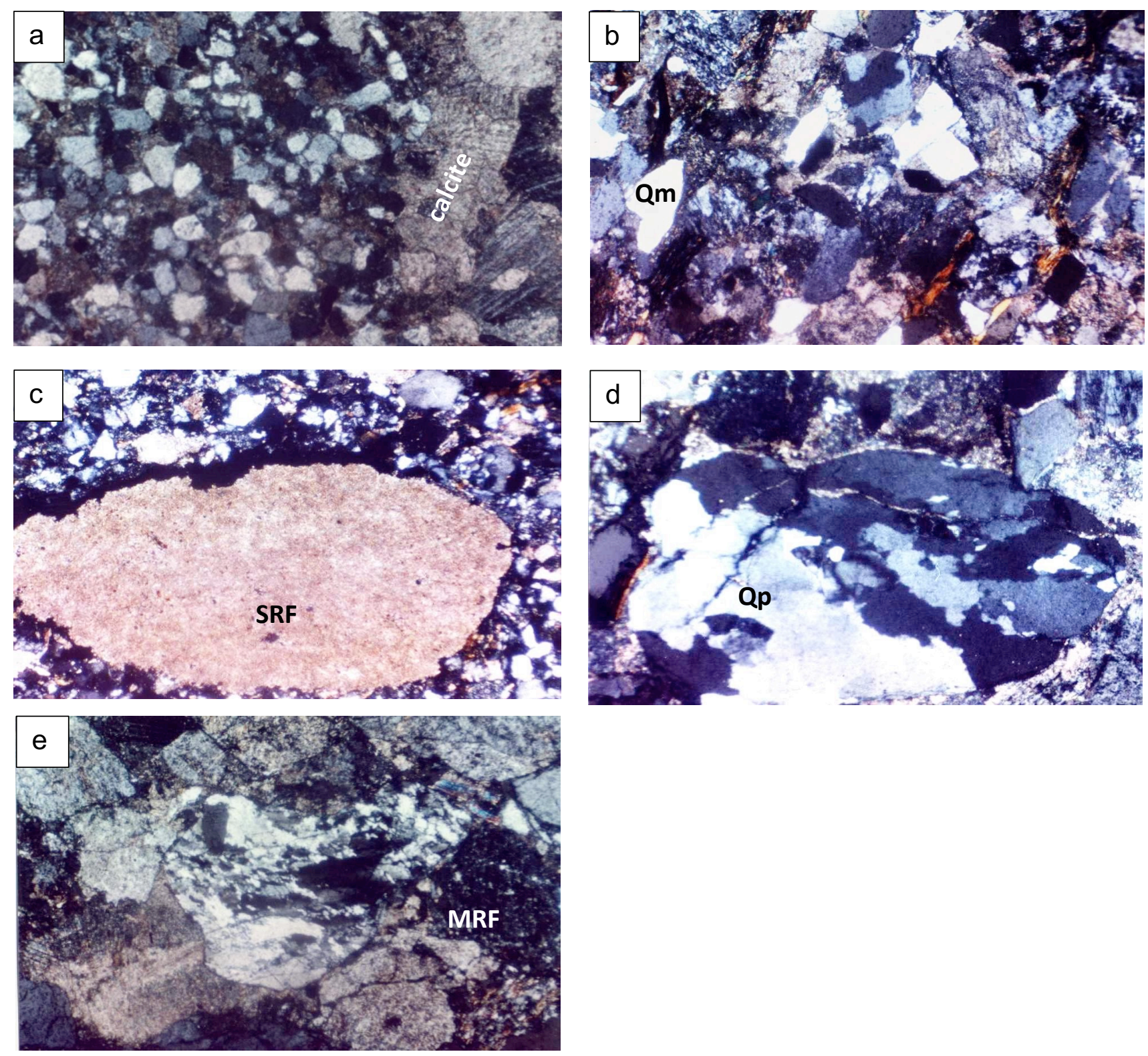

Fig. 5. Photomicrographs of sandstone samples of the Ispikan Conglomerate; all photographs are 40x and under crossed polarized light; (a) Medium- to fine-grained sandstone with diagenetically formed calcite; (b) Quartzose grains including Qm and Qp types; (c) Large mud chip (SRF) in medium- to fine-grained sandstone; (d) Qp granule showing granulated and crenulated crystallite - crystallite boundaries; (e) Metamorphic rock fragment having strongly undulose and stretched quartz showing typical crenulated crystal-crystal boundaries. 
Table 1. Point Counting data of Ispikan Conglomerate. Qmu; Undulose monocrystalline quartz, Qmnu; Non-uduolose monocrystalline quartz, Qpu: Undulose polycrystalline quartz, Qpnu; nonundulose polycrystalline quartz, K; Potassium feldspar, P; plagioclase feldspar, C; chert, SRF; sedimentary roks fragments, PRF; plutonic rock fragments, MRF; metamorphic rock fragments, VRF; volcanic rock fragments, Mus; musovite, Bio; biotite.

\begin{tabular}{|c|c|c|c|c|c|c|c|c|c|c|c|c|c|c|}
\hline & \multicolumn{14}{|c|}{ Percent Composition } \\
\hline Sample No. & Qmu & Qmnu & Qpu & Qpnu & $\mathrm{K}$ & $\mathrm{P}$ & $\mathrm{C}$ & SRF & PRF & MRF & VRF & Mus & Bio & Total \\
\hline ISP/A-1 & 11.6 & 37.9 & 8.1 & 5.1 & 7.6 & 5.6 & 0 & 2.5 & 0 & 7.6 & 10 & 4.0 & 0 & 100 \\
\hline ISP/A-3 & 60 & 40 & 0 & 0 & 0 & 0 & 0 & 0 & 0 & 0 & 0 & 0 & 0 & 100 \\
\hline ISP/A-4 & 60 & 40 & 0 & 0 & 0 & 0 & 0 & 0 & 0 & 0 & 0 & 0 & 0 & 100 \\
\hline ISP/A-14 & 0 & 20.8 & 13.0 & 6.5 & 10.4 & 13.0 & 6.5 & 10.4 & 3.9 & 10.4 & 5.1 & 0 & 0 & 100 \\
\hline ISP/A-16b & 70 & 0 & 30 & 0 & 0 & 0 & 0 & 0 & 0 & 0 & 0 & 0 & 0 & 100 \\
\hline ISP/A-17 & 12.0 & 18.0 & 10 & 12.0 & 6.0 & 10 & 8.0 & 6.0 & 2.0 & 8.0 & 8.0 & 0 & 0 & 100 \\
\hline ISP/A-18 & 23.5 & 41.9 & 2.9 & 6.6 & 11.0 & 8.1 & 0 & 0 & 0 & 0 & 3.8 & 0 & 2.2 & 100 \\
\hline ISP/A-19 & 14.6 & 43.8 & 8.3 & 6.3 & 7.6 & 5.6 & 1.4 & 4.9 & 1.4 & 3.4 & 2.7 & 0 & 0 & 100 \\
\hline ISP/B-2 & 9.9 & 76.4 & 2.9 & 0 & 5.8 & 3.3 & 1.7 & 0 & 0 & 0 & 0 & 0 & 0 & 100 \\
\hline ISP/B-4 & 0 & 27.3 & 3.6 & 7.3 & 3.6 & 1.8 & 0 & 21.8 & 0 & 7.3 & 27.3 & 0 & 0 & 100 \\
\hline ISP/B-5 & 18.1 & 26.3 & 7.6 & 9.9 & 8.8 & 4.1 & 1.2 & 5.8 & 7.6 & 6.4 & 4.2 & 0 & 0 & 100 \\
\hline ISP/B-6 & 13.9 & 25.0 & 5.6 & 12.5 & 8.3 & 11.1 & 1.4 & 6.9 & 5.5 & 5.6 & 4.2 & 0 & 0 & 100 \\
\hline ISP/B-7 & 18.6 & 25.6 & 7.0 & 10.5 & 7.6 & 5.8 & 1.2 & 5.2 & 7.6 & 6.8 & 4.1 & 0 & 0 & 100 \\
\hline ISP/B-8 & 21.6 & 35.1 & 5.4 & 8.1 & 8.1 & 10.8 & 0 & 5.4 & 5.5 & 0 & 0 & 0 & 0 & 100 \\
\hline ISP/B-12 & 21.4 & 29.8 & 11.9 & 17.9 & 2.4 & 7 & 0 & 2.4 & 2.4 & 2.4 & 2.4 & 0 & 0 & 100 \\
\hline ISP/B-13 & 0 & 63.4 & 0 & 12.2 & 0 & 14.7 & 0 & 2.4 & 0 & 2.4 & 4.9 & 0 & 0 & 100 \\
\hline ISP/B-14 & 0 & 34.3 & 7.1 & 17.1 & 7.2 & 7.1 & 0 & 10 & 2.9 & 2.9 & 11.4 & 0 & 0 & 100 \\
\hline ISP/B-15 & 19.7 & 14.8 & 6.6 & 4.9 & 16.4 & 8.2 & 0 & 6.6 & 9.8 & 8.1 & 3.3 & 1.6 & 0 & 100 \\
\hline ISP/B-21 & 25.9 & 15.5 & 12.9 & 11.2 & 7.8 & 12.9 & 0 & 2.6 & 3.4 & 4.3 & 1.7 & 0.9 & 0.9 & 100 \\
\hline ISP/B-22 & 15.2 & 43.9 & 9.1 & 2.4 & 6.4 & 9.8 & 1.8 & 3.0 & 1.8 & 2.4 & 1.8 & 1.2 & 1.2 & 100 \\
\hline ISP/B-23 & 13.1 & 32.8 & 13.9 & 8.8 & 3.7 & 10.9 & 0.7 & 5.8 & 5.1 & 2.2 & 1.5 & 0 & 1.5 & 100 \\
\hline ISP/B-24 & 0 & 62.0 & 0 & 10.9 & 9.7 & 7.6 & 0 & 3.3 & 2.2 & 4.3 & 0 & 0 & 0 & 100 \\
\hline ISP/B-25 & 12.6 & 21.1 & 13.7 & 15.8 & 5.3 & 10.5 & 1.1 & 3.2 & 5.3 & 7.1 & 2.1 & 1.1 & 1.1 & 100 \\
\hline ISP/B-26 & 0 & 37.6 & 12.9 & 7.5 & 16.2 & 14.0 & 0 & 8.6 & 0 & 3.2 & 0 & 0 & 0 & 100 \\
\hline ISP/B-27 & 6.3 & 55.6 & 3.2 & 9.5 & 0.7 & 11.9 & 4.0 & 2.4 & 0 & 4.8 & 1.6 & 0 & 0 & 100 \\
\hline ISP/C-2 & 17.0 & 39.0 & 6.7 & 7.8 & 3.6 & 10.6 & 0.7 & 3.5 & 4.3 & 2.5 & 2.5 & 0.4 & 1.4 & 100 \\
\hline ISP/C-3 & 0 & 52.9 & 1.3 & 0 & 9.4 & 12.9 & 0 & 23.5 & 0 & 0 & 0 & 0 & 0 & 100 \\
\hline ISP/C-8 & 52.1 & 36.5 & 3.1 & 8.3 & 0 & 0 & 0 & 0 & 0 & 0 & 0 & 0 & 0 & 100 \\
\hline ISP/P-1 & 32.1 & 15.1 & 7.5 & 5.1 & 6.3 & 9.4 & 0 & 3.1 & 9.4 & 1.9 & 6.3 & 1.3 & 2.5 & 100 \\
\hline ISP/P-3 & 12.7 & 41.4 & 6.6 & 6 & 4.4 & 6.6 & 0.6 & 1.7 & 3.9 & 6.1 & 7.2 & 2.2 & 0.6 & 100 \\
\hline ISP/P-5 & 16.2 & 37.9 & 6.5 & 7.4 & 3.6 & 9.7 & 0.7 & 2.9 & 3.6 & 2.5 & 7.2 & 0.4 & 1.4 & 100 \\
\hline ISP/P-7 & 16.2 & 37.9 & 6.7 & 7.2 & 3.6 & 9.7 & 0.7 & 2.9 & 3.6 & 2.5 & 7.2 & 0.4 & 1.4 & 100 \\
\hline ISP/B-10b & 21.8 & 36.4 & 6.1 & 4.1 & 6.7 & 7.9 & 0 & 6.1 & 2.4 & 7.9 & 0 & 0 & 0.6 & 100 \\
\hline ISP/P-23b & 0 & 100 & 0 & 0 & 0 & 0 & 0 & 0 & 0 & 0 & 0 & 0 & 0 & 100 \\
\hline ISP/WK-9 & 15.4 & 52.5 & 6.3 & 5.4 & 6.9 & 3.9 & 0 & 5.0 & 3.1 & 0 & 0 & 1.5 & 0 & 100 \\
\hline ISP/WK-17 & 6.1 & 28.0 & 10.5 & 7.9 & 7.9 & 6.1 & 0 & 6.7 & 7.9 & 6.1 & 10.4 & 1.8 & 0.6 & 100 \\
\hline ISP/WK-18 & 4.4 & 26.5 & 10.5 & 6.2 & 7.7 & 7.2 & 0 & 9.9 & 7.2 & 6.6 & 9.9 & 1.7 & 2.2 & 100 \\
\hline ISP/WK-19 & 12.6 & 25.2 & 7.6 & 5.9 & 10.9 & 2.5 & 1.7 & 10.9 & 1.7 & 9.2 & 11.8 & 0 & 0 & 100 \\
\hline ISP/WK-22 & 6.9 & 28.0 & 8.5 & 6.3 & 7.9 & 6.9 & 1.1 & 2.1 & 4.8 & 7.4 & 11.1 & 1.6 & 7.4 & 100 \\
\hline ISP/WK-23 & 9.1 & 12.3 & 7.3 & 5.8 & 11.7 & 8.4 & 5.8 & 6.5 & 7.8 & 9.7 & 9.1 & 3.9 & 2.6 & 100 \\
\hline
\end{tabular}


Spider diagram reveals that $\mathrm{Sr}, \mathrm{Rb}, \mathrm{V}$, and $\mathrm{Ba}$ show considerable variations whereas all other elements are the same in all samples (Figure 7). The categories of samples developed on the bases of major oxides and trace elements are tabulated in Table 2.

\section{Discussion}

Ispikan Conglomerate has a relatively small and insignificant exposure but bears considerable importance on the geological history of the region. Sedimentology, geochemistry and petrography of the Ispikan Conglomerate have provided several pieces of evidence to establish the composition of the source rocks and to reconstruct the paleogeographic conditions. Previously, only HSC (1960) speculated two possible sources based on large boulders and fragments of igneous rocks: a distant igneous exposure much farther east in Iran, and salt plugs on the analogy of salt plugs of Hormuz Strait. Both of these provenances are implausible because of the absence of any proximal igneous source or Salt Plugs. Kassi et al. (2007) proposed a new stratigraphic framework of Ispikan Conglomerate and other lithologic units of the area. They, however, did not comment on the provenance or mode of emplacement of Ispikan detritus.

We propose that the Ispikan Conglomerate is an olistostrome formed by mud-and-debris flow on the steep slope of continental margin - a common feature of the accretionary prism environment of the orogenic fronts (Burg et al., 2008; 2019; Heubeck, 1992; Cook, 1971; Renz, 1955). We present the following lines of arguments in support of our interpretation.

\subsection{Petrography}

The clastic mineral assemblage of the Ispikan Conglomerate indicates multiple source lithologies that provided sediments from the north-northwest of the present Ispikan. The angular to sub-angular poorly sorted, immature sandstone is indicative of short, traveled sediments from a proximal source. Poorly sorted and angular to sub-rounded framework grains i.e., Qm, Qp, F, RFs and heavy mineral grains also indicate short distance traveling of detritus. However, SRFs, on the other hand, have two types of populations, one type is well-rounded siltstone fragments which indicate far-traveled or rapid abrasion in a short time, whereas the second population is composed of very angular mud chips, very angular fragments of sparry calcite and slightly abraded but mostly complete fossil fragments. The second population of grains is intrabasinal that have been supplied by the nearby carbonate rocks (Eocene Wakai Limestone and equivalent) that were present close to the depositional site.

The presence of granitoid, andesitic and metamorphic clasts reflect the presence of plutonic and volcanic terrane in the source area. Contact metamorphism is associated with plutonism and volcanism, therefore it is common to have metamorphic rock fragments from volcanic and plutonic sources. Granitoid and metamorphic clasts are very angular to sub-angular whereas andesitic clasts are mostly well-rounded showing distant traveling. 


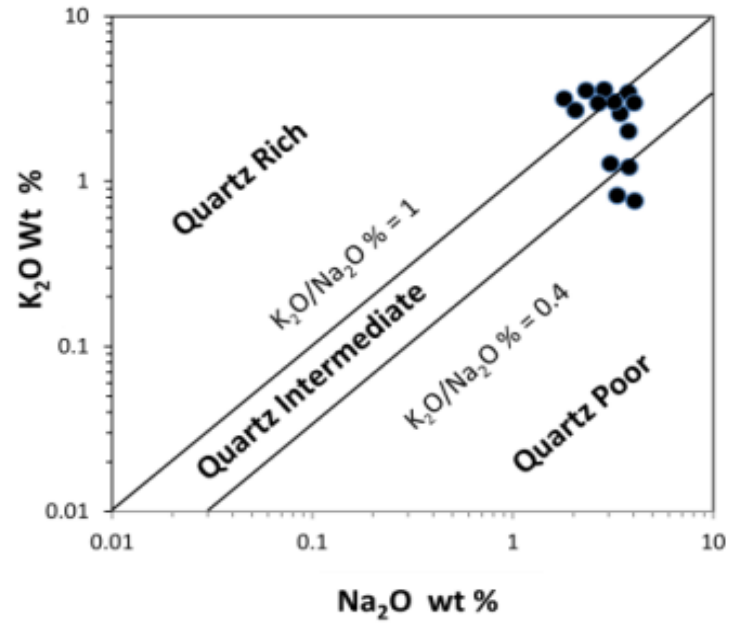

Fig. 6. $\mathrm{K}_{2} \mathrm{O} \mathrm{Vs} \mathrm{Na}_{2} \mathrm{O}$ classification of the sandstone fraction of Ispikan Conglomerate (Crook 1974).
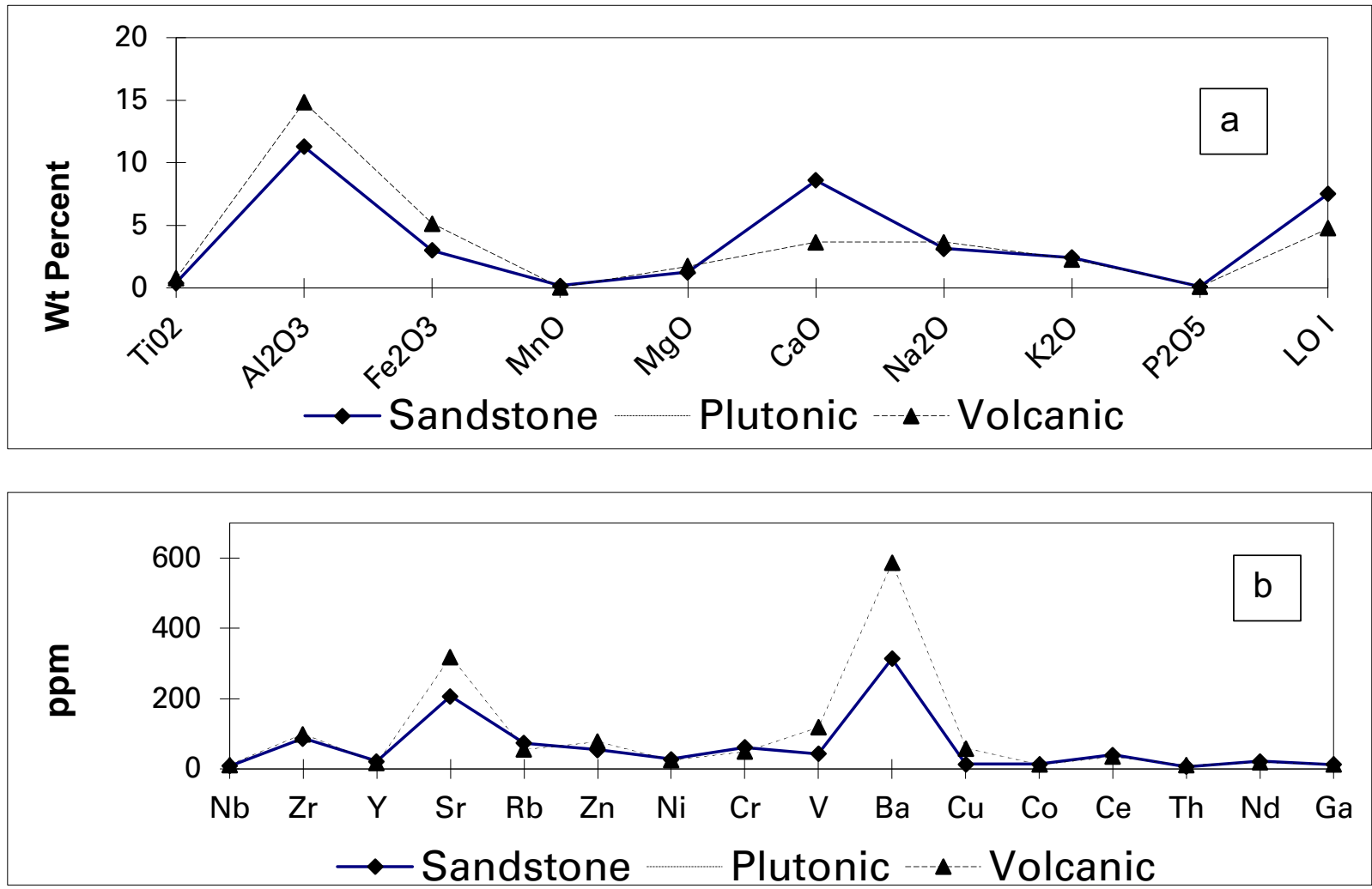

Fig. 7. Spider diagram showing distribution and composition of average concentration of (a) major oxides (except $\mathrm{SiO}_{2}$ ), (b) average amount of trace elements in sandstone samples and igneous pebbles from Ispikan Conglomerate. 


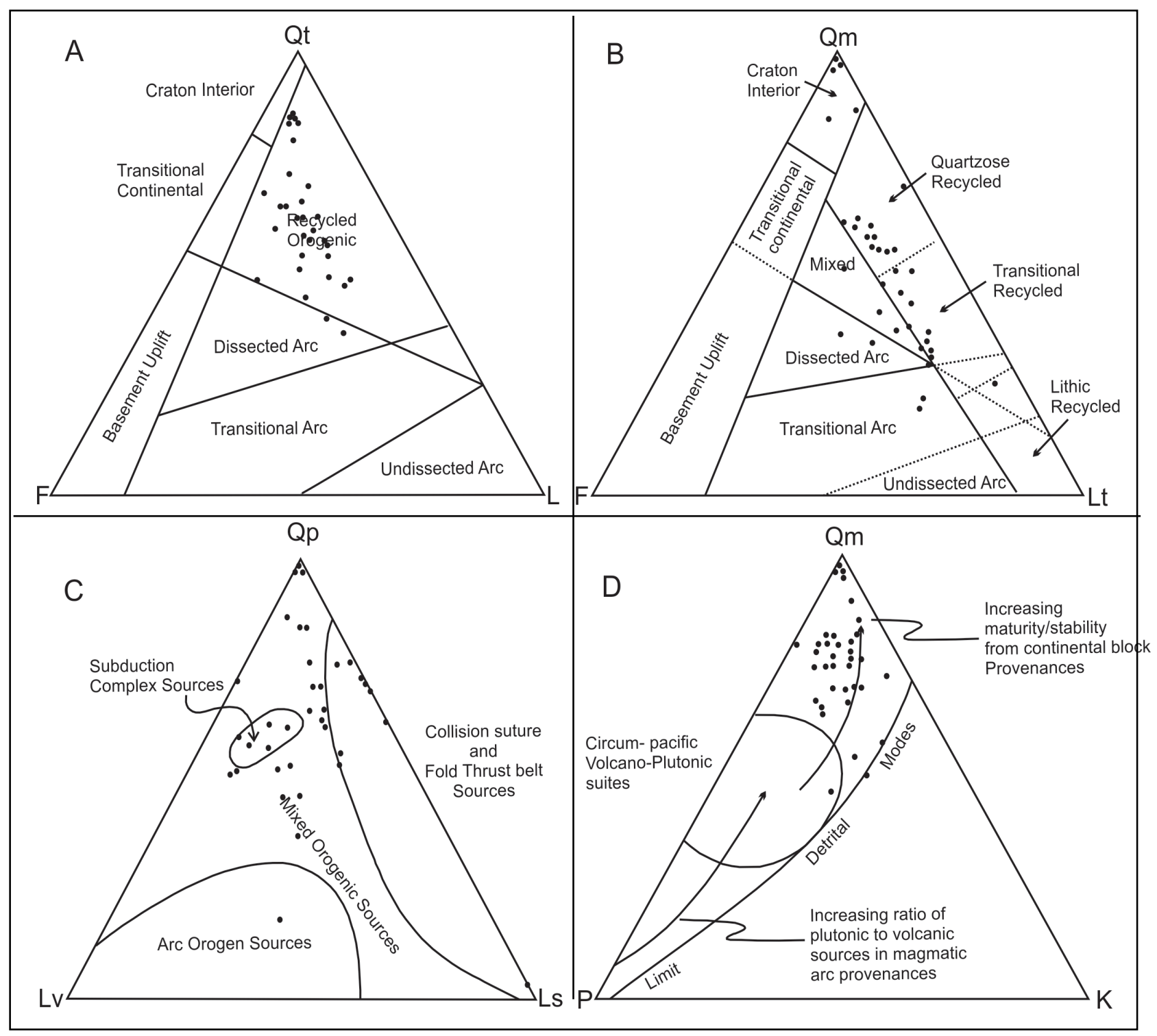

Fig. 8. Provenance discrimination ternary diagrams of Ispikan Conglomerate (after Dickinson and Suzek 1979). Qt; total quartz, F; feldspar, Lt; total lithics, Qm; monocrystalline quartz, Qp; polycrystalline quartz, P; plagioclase feldspar, K, potassium feldspar, Lv; volcanic lithics, Ls; sedimentary lithics. 
Table 2. Major and trace element concentrations in the sediments of Ispikan Conglomerate, Makran Accretionary Prism, Southwest Pakistan. BDL; Below detection limit.

\begin{tabular}{|c|c|c|c|c|c|c|c|c|c|}
\hline Major Oxides (Wt \%) & ISP- A1 & ISP-A14 & ISP- A17 & ISP- A18 & ISP-A19 & ISP-B2 & ISP-B6 & ISP-B7 & ISP-B8 \\
\hline $\mathrm{SiO}_{2}$ & 57.1 & 59.4 & 75.1 & 77.4 & 63.4 & 65.7 & 66.2 & 61.5 & 64.0 \\
\hline $\mathrm{TiO}_{2}$ & 0.3 & 0.3 & 0.2 & 1.1 & 1.2 & 0.1 & 0.4 & 0.1 & 0.4 \\
\hline $\mathrm{Al}_{2} \mathrm{O}_{3}$ & 11.7 & 11.6 & 11.3 & 11.2 & 10.2 & 10.1 & 14.9 & 8.4 & 15.0 \\
\hline $\mathrm{Fe}_{2} \mathrm{O}_{3}$ & 1.9 & 2.5 & 1.6 & 2.2 & 1.2 & 1.7 & 5.9 & 1.6 & 5.5 \\
\hline $\mathrm{MnO}$ & 0.2 & 0.3 & 0 & 0.1 & 0.1 & 0.2 & 0.2 & 0.3 & 0 \\
\hline $\mathrm{MgO}$ & 0.8 & 0.7 & 0.7 & 0.6 & 0.5 & 0.3 & 2.0 & 0.2 & 2.1 \\
\hline $\mathrm{CaO}$ & 11.7 & 10.6 & 2.2 & 1.1 & 10.1 & 9.0 & 1.2 & 12.9 & 2.1 \\
\hline $\mathrm{Na}_{2} \mathrm{O}$ & 3.8 & 3.4 & 3.2 & 2.9 & 2.7 & 2.3 & 3.8 & 1.8 & 4.0 \\
\hline $\mathrm{K}_{2} \mathrm{O}$ & 2.0 & 2.6 & 3.0 & 3.6 & 3.0 & 3.5 & 3.5 & 3.2 & 3.0 \\
\hline $\mathrm{P}_{2} \mathrm{O}_{5}$ & 0.1 & 0.2 & 0.1 & 0.1 & 0.1 & 0.1 & 0.1 & 0.1 & 0.1 \\
\hline LOl & 10.3 & 8.5 & 2.6 & 0.7 & 8.7 & 6.8 & 1.9 & 10 & 3.6 \\
\hline Total & 99.9 & 99.9 & 100 & 100.9 & 101.0 & 100 & 99.9 & 100 & 100 \\
\hline \multicolumn{10}{|c|}{ Trace Elements (ppm) } \\
\hline $\mathrm{Nb}$ & 6 & 8 & 5 & 7 & 5 & 9 & 13 & 9 & 9 \\
\hline $\mathrm{Zr}$ & 90 & 70 & 86 & 66 & 77 & 52 & 105 & 50 & 130 \\
\hline $\mathrm{Y}$ & 25 & 29 & 14 & 18 & 20 & 24 & 22 & 26 & 18 \\
\hline $\mathrm{Sr}$ & 306 & 278 & 166 & 138 & 141 & 113 & 144 & 123 & 172 \\
\hline $\mathrm{Rb}$ & 66 & 81 & 87 & 102 & 86 & 101 & 102 & 88 & 87 \\
\hline $\mathrm{Zn}$ & 35 & 48 & 28 & 41 & 21 & 34 & 112 & 32 & 99 \\
\hline $\mathrm{Ni}$ & 12 & 17 & 11 & 16 & 6 & 13 & 28 & 13 & 21 \\
\hline $\mathrm{Cr}$ & 27 & 25 & 16 & 24 & 22 & 30 & 43 & 105 & 35 \\
\hline $\mathrm{V}$ & 40 & 45 & 10 & 15 & 8 & 13 & 68 & 0.01 & 63 \\
\hline $\mathrm{Ba}$ & 289 & 280 & 544 & 535 & 364 & 355 & 401 & 436 & 410 \\
\hline $\mathrm{Cu}$ & 11 & 14 & 8 & 11 & 5 & 8 & 21 & 10 & 18 \\
\hline Co & 14 & 10 & 7 & BDL & 8 & 4 & 4 & 5 & 8 \\
\hline $\mathrm{Ce}$ & 49 & 42 & 22 & 15 & 29 & 23 & 42 & 55 & 48 \\
\hline $\mathrm{Th}$ & 5 & 8 & 8 & 11 & 5 & 2 & 5 & 3 & 8 \\
\hline $\mathrm{Nd}$ & 27 & 30 & 14 & 17 & 20 & 23 & 34 & 19 & 31 \\
\hline $\mathrm{Ga}$ & 10 & 14 & 10 & 14 & 9 & 13 & 19 & 12 & 15 \\
\hline \multicolumn{10}{|c|}{ Recalculated parameters } \\
\hline $\mathrm{Al}_{2} \mathrm{O}_{3} / \mathrm{SiO}_{2}$ & 0.20 & 0.19 & 0.15 & 0.14 & 0.16 & 0.15 & 0.22 & 0.14 & 0.23 \\
\hline $\mathrm{Al}_{2} \mathrm{O}_{3} /\left(\mathrm{CaO}+\mathrm{Na}_{2} \mathrm{O}\right)$ & 0.76 & 0.82 & 2.09 & 2.80 & 0.80 & 0.89 & 3.01 & 0.57 & 2.44 \\
\hline $\mathrm{Fe}_{2} \mathrm{O}_{3}+\mathrm{MgO}$ & 2.71 & 3.14 & 2.35 & 2.78 & 1.62 & 2.05 & 7.93 & 1.82 & 7.62 \\
\hline $\mathrm{Na}_{2} \mathrm{O}+\mathrm{K}_{2} \mathrm{O}$ & 5.79 & 6 & 6.23 & 6.44 & 5.65 & 5.86 & 7.23 & 4.96 & 7.02 \\
\hline $\mathrm{K}_{2} \mathrm{O} / \mathrm{Na}_{2} \mathrm{O}$ & 0.53 & 0.75 & 0.95 & 1.26 & 1.12 & 1.53 & 0.91 & 1.76 & 0.74 \\
\hline $\mathrm{SiO}_{2} / \mathrm{Al}_{2} \mathrm{O}_{3}$ & 4.88 & 5.14 & 6.65 & 6.94 & 6.22 & 6.53 & 4.45 & 7.35 & 4.27 \\
\hline $\mathrm{Fe}_{2} \mathrm{O}_{3} / \mathrm{MgO}$ & 2.47 & 3.82 & 2.26 & 3.71 & 2.52 & 5.20 & 2.98 & 6.43 & 2.56 \\
\hline $\mathrm{Zr} / \mathrm{Y}$ & 3.60 & 2.41 & 6.14 & 3.67 & 3.85 & 2.17 & 4.77 & 1.92 & 7.22 \\
\hline $\mathrm{Zr} / \mathrm{Th}$ & 18 & 8.75 & 10.75 & 6 & 15.40 & 26 & 21 & 16.67 & 16.25 \\
\hline $\mathrm{Nb} / \mathrm{Y}$ & 0.24 & 0.28 & 0.36 & 0.39 & 0.25 & 0.38 & 0.59 & 0.35 & 0.50 \\
\hline $\mathrm{Y}+\mathrm{Nb}$ & 31 & 37 & 19 & 25 & 25 & 33 & 35 & 35 & 27 \\
\hline $\mathrm{Ba} / \mathrm{Y}$ & 11.56 & 9.66 & 38.86 & 29.72 & 18.20 & 14.79 & 18.23 & 16.77 & 22.78 \\
\hline $\mathrm{Zr} / \mathrm{Nb}$ & 15 & 8.75 & 17.20 & 9.43 & 15.40 & 5.78 & 8.08 & 5.56 & 14.44 \\
\hline $\mathrm{Rb} / \mathrm{Sr}$ & 0.22 & 0.29 & 0.52 & 0.74 & 0.61 & 0.89 & 0.71 & 0.72 & 0.51 \\
\hline $\mathrm{Ba} / \mathrm{Sr}$ & 0.94 & 1.01 & 3.28 & 3.88 & 2.58 & 3.14 & 2.78 & 3.54 & 2.38 \\
\hline
\end{tabular}




\begin{tabular}{|c|c|c|c|c|c|c|c|c|c|}
\hline Major Oxides (Wt\%) & ISP-B12 & ISP-B24 & ISP-P5 & WK-9 & WK-18 & ISP - A7 & ISP-A11 & ISP-C9 & ISP-C 10 \\
\hline $\mathrm{SiO}_{2}$ & 51.7 & 59.3 & 61.2 & 49.5 & 59.0 & 71.2 & 74.7 & 71.9 & 70.9 \\
\hline $\mathrm{TiO}_{2}$ & 0.4 & 0.1 & 0.4 & 0.4 & 0.4 & 0.2 & 0.1 & 0.2 & 0.2 \\
\hline $\mathrm{Al}_{2} \mathrm{O}_{3}$ & 12.9 & 8.5 & 9.7 & 13.0 & 9.8 & 16.0 & 14.2 & 16.3 & 15.8 \\
\hline $\mathrm{Fe}_{2} \mathrm{O}_{3}$ & 5.3 & 1.1 & 3.6 & 4.9 & 3.1 & 3.0 & 1.0 & 3.3 & 3.5 \\
\hline $\mathrm{MnO}$ & 0.3 & 0.2 & 0.3 & 0.2 & 0.1 & 0 & 0 & 0.1 & 0 \\
\hline $\mathrm{MgO}$ & 2.9 & 0.4 & 1.6 & 3.1 & 1.7 & 0.4 & 0.2 & 0.2 & 0.4 \\
\hline $\mathrm{CaO}$ & 11.3 & 13.9 & 10.4 & 12.3 & 11.4 & 0.4 & 0.5 & 0.2 & 0.3 \\
\hline $\mathrm{Na}_{2} \mathrm{O}$ & 3.8 & 2.1 & 3.1 & 4.1 & 3.3 & 2.0 & 3.0 & 2.1 & 0.8 \\
\hline $\mathrm{K}_{2} \mathrm{O}$ & 1.2 & 2.7 & 1.3 & 0.8 & 0.8 & 5.6 & 5.1 & 4.5 & 6.8 \\
\hline $\mathrm{P}_{2} \mathrm{O}_{5}$ & 0.1 & 0.1 & 0.1 & 0.1 & 0.1 & 0.3 & 0.3 & 0.3 & 0.3 \\
\hline Lol & 10.1 & 11.7 & 8.5 & 11.8 & 10.2 & 0.8 & 0.8 & 0.9 & 0.9 \\
\hline Total & 100 & 100 & 100 & 100 & 100 & 100 & 100 & 100 & 99.9 \\
\hline \multicolumn{10}{|c|}{ Trace Elements (ppm) } \\
\hline $\mathrm{Nb}$ & 14 & 5 & 11 & 10 & 7 & 20 & 14 & 42 & 15 \\
\hline $\mathrm{Zr}$ & 97 & 75 & 87 & 120 & 110 & 47 & 45 & 19 & 36 \\
\hline $\mathrm{Y}$ & 27 & 22 & 23 & 23 & 19 & 14 & 10 & 8 & 12 \\
\hline $\mathrm{Sr}$ & 261 & 151 & 292 & 287 & 318 & 44 & 41 & 12 & 57 \\
\hline $\mathrm{Rb}$ & 47 & 73 & 46 & 32 & 31 & 267 & 261 & 426 & 304 \\
\hline $\mathrm{Zn}$ & 99 & 19 & 57 & 86 & 44 & 23 & 18 & 27 & 21 \\
\hline $\mathrm{Ni}$ & 61 & 6 & 67 & 54 & 60 & 11 & 4 & 13 & 15 \\
\hline $\mathrm{Cr}$ & 52 & 97 & 171 & 44 & 163 & 15 & 12 & 50 & 153 \\
\hline $\mathrm{V}$ & 103 & 0.01 & 71 & 99 & 67 & $\mathrm{BDL}$ & $\mathrm{BDL}$ & 16 & BDL \\
\hline $\mathrm{Ba}$ & 18 & 445 & 157 & 9 & 148 & 116 & 111 & 36 & 234 \\
\hline $\mathrm{Cu}$ & 27 & 7 & 17 & 24 & 14 & 12 & 6 & 11 & 12 \\
\hline $\mathrm{Co}$ & 35 & 9 & 21 & 39 & 25 & 12 & 9 & 9 & 13 \\
\hline $\mathrm{Ce}$ & 33 & 61 & 46 & 39 & 52 & 26 & 22 & 20 & 13 \\
\hline Th & 5 & 6 & 5 & 7 & 7 & 12 & 6 & 9 & 9 \\
\hline $\mathrm{Nd}$ & 20 & 16 & 25 & 7 & 22 & 13 & 6 & 22 & 15 \\
\hline $\mathrm{Ga}$ & 14 & 8 & 13 & 10 & 9 & 22 & 19 & 34 & 21 \\
\hline \multicolumn{10}{|c|}{ Recalculated parameters } \\
\hline $\mathrm{Al}_{2} \mathrm{O}_{3} / \mathrm{SiO}_{2}$ & 0.25 & 0.14 & 0.16 & 0.26 & 0.17 & 0.23 & 0.19 & 0.23 & 0.22 \\
\hline $\mathrm{Al}_{2} \mathrm{O}_{3} /\left(\mathrm{CaO}+\mathrm{Na}_{2} \mathrm{O}\right)$ & 0.85 & 0.53 & 0.72 & 0.80 & 0.67 & 6.66 & 4.03 & 7.04 & 14.67 \\
\hline $\mathrm{Fe}_{2} \mathrm{O}_{3}+\mathrm{MgO}$ & 8.22 & 1.51 & 5.12 & 7.91 & 4.81 & 3.40 & 1.25 & 3.57 & 3.88 \\
\hline $\mathrm{Na}_{2} \mathrm{O}+\mathrm{K}_{2} \mathrm{O}$ & 5.02 & 4.75 & 4.35 & 4.81 & 4.14 & 7.64 & 8.06 & 6.67 & 7.65 \\
\hline $\mathrm{K}_{2} \mathrm{O} / \mathrm{Na}_{2} \mathrm{O}$ & 0.32 & 1.32 & 0.42 & 0.19 & 0.25 & 2.80 & 1.70 & 2.13 & 8.33 \\
\hline $\mathrm{SiO}_{2} / \mathrm{Al}_{2} \mathrm{O}_{3}$ & 4.02 & 6.98 & 6.31 & 3.81 & 6 & 4.44 & 5.26 & 4.40 & 4.48 \\
\hline $\mathrm{Fe}_{2} \mathrm{O}_{3} / \mathrm{MgO}$ & 1.83 & 2.87 & 2.29 & 1.59 & 1.83 & 8.44 & 4.43 & 14.52 & 8.02 \\
\hline $\mathrm{Zr} / \mathrm{Y}$ & 3.59 & 3.41 & 3.78 & 5.22 & 5.79 & 3.36 & 4.50 & 2.38 & 3 \\
\hline $\mathrm{Zr} / \mathrm{Th}$ & 19.40 & 12.50 & 17.40 & 17.14 & 15.71 & 3.92 & 7.50 & 2.11 & 4 \\
\hline $\mathrm{Nb} / \mathrm{Y}$ & 0.52 & 0.23 & 0.48 & 0.43 & 0.37 & 1.43 & 1.40 & 5.25 & 1.25 \\
\hline $\mathrm{Y}+\mathrm{Nb}$ & 41 & 27 & 34 & 33 & 26 & 34 & 24 & 50 & 27 \\
\hline $\mathrm{Ba} / \mathrm{Y}$ & 0.67 & 20.23 & 6.83 & 0.39 & 7.79 & 8.29 & 11.10 & 4.50 & 19.50 \\
\hline $\mathrm{Zr} / \mathrm{Nb}$ & 6.93 & 15 & 7.91 & 12 & 15.71 & 2.35 & 3.21 & 0.45 & 2.40 \\
\hline $\mathrm{Rb} / \mathrm{Sr}$ & 0.18 & 0.48 & 0.16 & 0.11 & 0.10 & 6.07 & 6.37 & 35.50 & 5.33 \\
\hline $\mathrm{Ba} / \mathrm{Sr}$ & 0.07 & 2.95 & 0.54 & 0.03 & 0.47 & 2.64 & 2.71 & 3 & 4.11 \\
\hline
\end{tabular}




\begin{tabular}{|c|c|c|c|c|c|c|c|c|c|c|}
\hline Major Oxides (Wt\%) & ISP-X1 & ISP-X2 & ISP-X3 & ISP-X4 & ISP-A5 & ISP-A12 & ISP-B16 & ISP-C5 & ISP-E1 & ISP-P21 \\
\hline $\mathrm{SiO}_{2}$ & 75.6 & 74.7 & 62.3 & 66.0 & 74.2 & 44.7 & 41.0 & 77.9 & 71.5 & 67.8 \\
\hline $\mathrm{TiO}_{2}$ & 0.1 & 0.1 & 0.8 & 0.7 & 0.2 & 1.7 & 1.7 & 0.2 & 0.4 & 0.5 \\
\hline $\mathrm{Al}_{2} \mathrm{O}_{3}$ & 14.4 & 13.9 & 17.3 & 15.4 & 13.5 & 15.8 & 17.7 & 11.6 & 14.2 & 16.1 \\
\hline $\mathrm{Fe}_{2} \mathrm{O}_{3}$ & 1.3 & 1.4 & 7.6 & 5.5 & 3.4 & 9.1 & 11.2 & 1.3 & 1.4 & 4.4 \\
\hline $\mathrm{MnO}$ & 0.1 & 0.0 & 0.1 & 0.1 & 0 & 0.1 & 0.1 & 0 & 0 & 0 \\
\hline $\mathrm{MgO}$ & 0.1 & 0.3 & 2.0 & 1.8 & 0.7 & 3.4 & 3.6 & 0.6 & 1.0 & 1.1 \\
\hline $\mathrm{CaO}$ & 0.3 & 0.4 & 2.5 & 2.7 & 0.2 & 9.2 & 9.1 & 0.4 & 1.6 & 1.5 \\
\hline $\mathrm{Na}_{2} \mathrm{O}$ & 3.1 & 1.8 & 1.6 & 2.6 & 2.3 & 5.1 & 4.1 & 3.3 & 4.1 & 3.2 \\
\hline $\mathrm{K}_{2} \mathrm{O}$ & 4.0 & 6.3 & 4.5 & 3.9 & 3.9 & 0.7 & 1.3 & 3.3 & 2.0 & 2.6 \\
\hline $\mathrm{P}_{2} \mathrm{O}_{5}$ & 0.2 & 0.2 & 0.2 & 0.2 & 0.1 & 0.1 & 0.2 & 0 & 0.1 & 0.2 \\
\hline LOl & 0.9 & 0.9 & 1.1 & 1.2 & 1.5 & 10.1 & 10.1 & 1.5 & 2.8 & 2.7 \\
\hline Total & 100 & 100 & 100 & 100 & 100 & 100 & 100.1 & 100 & 99.1 & 100.1 \\
\hline \multicolumn{11}{|c|}{ Trace Elements (ppm) } \\
\hline $\mathrm{Nb}$ & 36 & 9 & 20 & 14 & 17 & 7 & 13 & 11 & 8 & 14 \\
\hline $\mathrm{Zr}$ & 17 & 34 & 170 & 168 & 111 & 78 & 80 & 109 & 108 & 110 \\
\hline $\mathrm{Y}$ & 4 & 8 & 49 & 45 & 20 & 22 & 26 & 16 & 7 & 11 \\
\hline $\mathrm{Sr}$ & 9 & 54 & 214 & 211 & 186 & 649 & 652 & 183 & 121 & 124 \\
\hline $\mathrm{Rb}$ & 420 & 298 & 159 & 153 & 72 & 22 & 28 & 66 & 69 & 75 \\
\hline $\mathrm{Zn}$ & 22 & 16 & 76 & 71 & 29 & 145 & 150 & 24 & 58 & 63 \\
\hline $\mathrm{Ni}$ & 6 & 8 & 28 & 21 & 11 & 32 & 39 & 4 & 27 & 34 \\
\hline $\mathrm{Cr}$ & 47 & 150 & 72 & 69 & 12 & 78 & 81 & 9 & 56 & 59 \\
\hline $\mathrm{V}$ & $\mathrm{BDL}$ & BDL & 126 & 122 & BDL & 301 & 305 & BDL & 51 & 55 \\
\hline $\mathrm{Ba}$ & 31 & 229 & 516 & 511 & 1456 & 195 & 200 & 1451 & 104 & 109 \\
\hline $\mathrm{Cu}$ & 5 & 6 & 10 & 4 & 10 & 156 & 162 & 4 & 5 & 11 \\
\hline $\mathrm{Co}$ & 6 & 10 & 20 & 17 & 4 & 21 & 24 & 1 & 11 & 14 \\
\hline $\mathrm{Ce}$ & 16 & 9 & 47 & 43 & 64 & 24 & 28 & 60 & 15 & 19 \\
\hline Th & 3 & 3 & 20 & 14 & 18 & 4 & 10 & 12 & 6 & 12 \\
\hline $\mathrm{Nd}$ & 15 & 8 & 34 & 27 & 25 & 16 & 23 & 18 & 11 & 18 \\
\hline $\mathrm{Ga}$ & 31 & 18 & 23 & 20 & 11 & 14 & 17 & 8 & 14 & 17 \\
\hline \multicolumn{11}{|c|}{ Recalculated parameters } \\
\hline $\mathrm{Al}_{2} \mathrm{O}_{3} / \mathrm{SiO}_{2}$ & 0.19 & 0.19 & 0.28 & 0.23 & 0.18 & 0.35 & 0.43 & 0.15 & 0.20 & 0.24 \\
\hline $\mathrm{Al}_{2} \mathrm{O}_{3} /\left(\mathrm{CaO}+\mathrm{Na}_{2} \mathrm{O}\right)$ & 4.20 & 6.35 & 4.20 & 2.94 & 5.37 & 1.11 & 1.35 & 3.20 & 2.48 & 3.49 \\
\hline $\mathrm{Fe}_{2} \mathrm{O}_{3}+\mathrm{MgO}$ & 1.36 & 1.67 & 9.51 & 7.30 & 4.10 & 12.54 & 14.75 & 1.89 & 2.37 & 5.58 \\
\hline $\mathrm{Na}_{2} \mathrm{O}+\mathrm{K}_{2} \mathrm{O}$ & 7.09 & 8.07 & 6.10 & 6.52 & 6.14 & 5.79 & 5.37 & 6.56 & 6.14 & 5.72 \\
\hline $\mathrm{K}_{2} \mathrm{O} / \mathrm{Na}_{2} \mathrm{O}$ & 1.28 & 3.48 & 2.81 & 1.53 & 1.69 & 0.15 & 0.32 & 1.01 & 0.48 & 0.81 \\
\hline $\mathrm{SiO}_{2} / \mathrm{Al}_{2} \mathrm{O}_{3}$ & 5.25 & 5.37 & 3.59 & 4.29 & 5.48 & 2.83 & 2.31 & 6.72 & 5.04 & 4.20 \\
\hline $\mathrm{Fe}_{2} \mathrm{O}_{3} / \mathrm{MgO}$ & 12.60 & 4.57 & 3.88 & 3.01 & 4.77 & 2.66 & 3.14 & 2.26 & 1.35 & 3.89 \\
\hline $\mathrm{Zr} / \mathrm{Y}$ & 4.25 & 4.25 & 3.47 & 3.73 & 5.55 & 3.55 & 3.08 & 6.81 & 15.43 & 10 \\
\hline $\mathrm{Zr} / \mathrm{Th}$ & 5.67 & 11.33 & 8.50 & 12 & 6.17 & 19.50 & 8 & 9.08 & 18 & 9.17 \\
\hline $\mathrm{Nb} / \mathrm{Y}$ & 9 & 1.13 & 0.41 & 0.31 & 0.85 & 0.32 & 0.50 & 0.69 & 1.14 & 1.27 \\
\hline $\mathrm{Y}+\mathrm{Nb}$ & 40 & 17 & 69 & 59 & 37 & 29 & 39 & 27 & 15 & 25 \\
\hline $\mathrm{Ba} / \mathrm{Y}$ & 7.75 & 28.63 & 10.53 & 11.36 & 72.80 & 8.86 & 7.69 & 90.69 & 14.86 & 9.91 \\
\hline $\mathrm{Zr} / \mathrm{Nb}$ & 0.47 & 3.78 & 8.50 & 12 & 6.53 & 11.14 & 6.15 & 9.91 & 13.50 & 7.86 \\
\hline $\mathrm{Rb} / \mathrm{Sr}$ & 46.67 & 5.52 & 0.74 & 0.73 & 0.39 & 0.03 & 0.04 & 0.36 & 0.57 & 0.60 \\
\hline $\mathrm{Ba} / \mathrm{Sr}$ & 3.44 & 4.24 & 2.41 & 2.42 & 7.83 & 0.30 & 0.31 & 7.93 & 0.86 & 0.88 \\
\hline
\end{tabular}




\subsection{Modal Analysis}

In the QFL diagrams of Dickinson $(1985 ; 1988)$, the samples show their affiliation with the Recycled Orogen and Continental Block provenance (Figure 8). According to Dickinson and Suczek (1979) sediments plotting within the continental block provenance are derived either from stable shields and platforms or from areas of uplift. In the Qm-F-Lt diagram most of the Ispikan samples plot in the quartzose and transitional recycled area that indicate their derivation form cratonic source. Almost all the samples plot outside the two delimited fields indicating that the source area was neither a collisional nor an arc orogen exclusively, however, it may have been a mixed orogenic provenance. In the Qm-P-K plot, the samples indicate Continental Block Provenance with an increasing trend towards maturity (i.e., Qm).

\subsection{Geochemistry}

A wide range of geochemical approaches are being routinely used for discriminating the tectonic environment of the detritus (Huang et al., 2018). Ispikan samples are classified as quartzintermediate based on the $\mathrm{K}_{2} \mathrm{O} / \mathrm{Na}_{2} \mathrm{O}$ ratio, indicating their derivation from the tectonically active continental margin on or adjacent to active plate boundaries (Crook, 1974). Similarly, Bhatia (1983), devised a series of plots to differentiate four main tectonic settings namely passive margin (PM), active continental margin (ACM), continental island arc (CIA), and oceanic island arc (OIC). The Ispikan samples are scattered across three fields of CIA, ACM, and PM (Figure 9) indicating heterogeneous provenance. A similar interpretation has been put forward by Aziz and Sadiq (2020) for the upper Cretaceous Tanjero Flysch of Northeast Iraq.

Multiple element discriminant function diagrams of Bhatia (1983) are also considered useful for discriminating tectonic settings. Most of the samples fall in Felsic igneous and intermediate igneous provenances (Figure 10). Function-1 of Bhatia (1983) has a high influence on $\mathrm{CaO}$ and $\mathrm{Na}_{2} \mathrm{O}$ while Function-2 is primarily influenced by $\mathrm{SiO}_{2}$ and $\mathrm{CaO}$. Sedimentary processes readily cause changes in the concentrations of $\mathrm{Na}$ and $\mathrm{Ca}$ owing to their high mobility. Lower concentrations of $\mathrm{Na}$ and $\mathrm{Ca}$ indicate felsic igneous provenance. Additionally, the scatter of data points in (Figure 11 ) is also because of moderate sorting and heterogeneity of the samples

Very high concentrations of $\mathrm{Cr}$ and $\mathrm{Ni}$ have been used to indicate an ultramafic provenance (Hiscott, 1984; Haughton, 1988; Wrafter \& Graham, 1989). The low levels of Cr (16-171 ppm) and Ni (6-67 ppm) recorded in the Ispikan detritus suggest either some basic input into the system or else that the trace elements could have travelled into the depositional basin as adsorbed ions on clays (McCann, 1991). Vanadium levels are relatively higher (up to $103 \mathrm{ppm}$ ) than the levels commonly recorded in sandstones (20 ppm) and given that $\mathrm{V}$ is more concentrated in basic rocks it suggests input from basic rocks into the depositional system (McCann, 1991). 

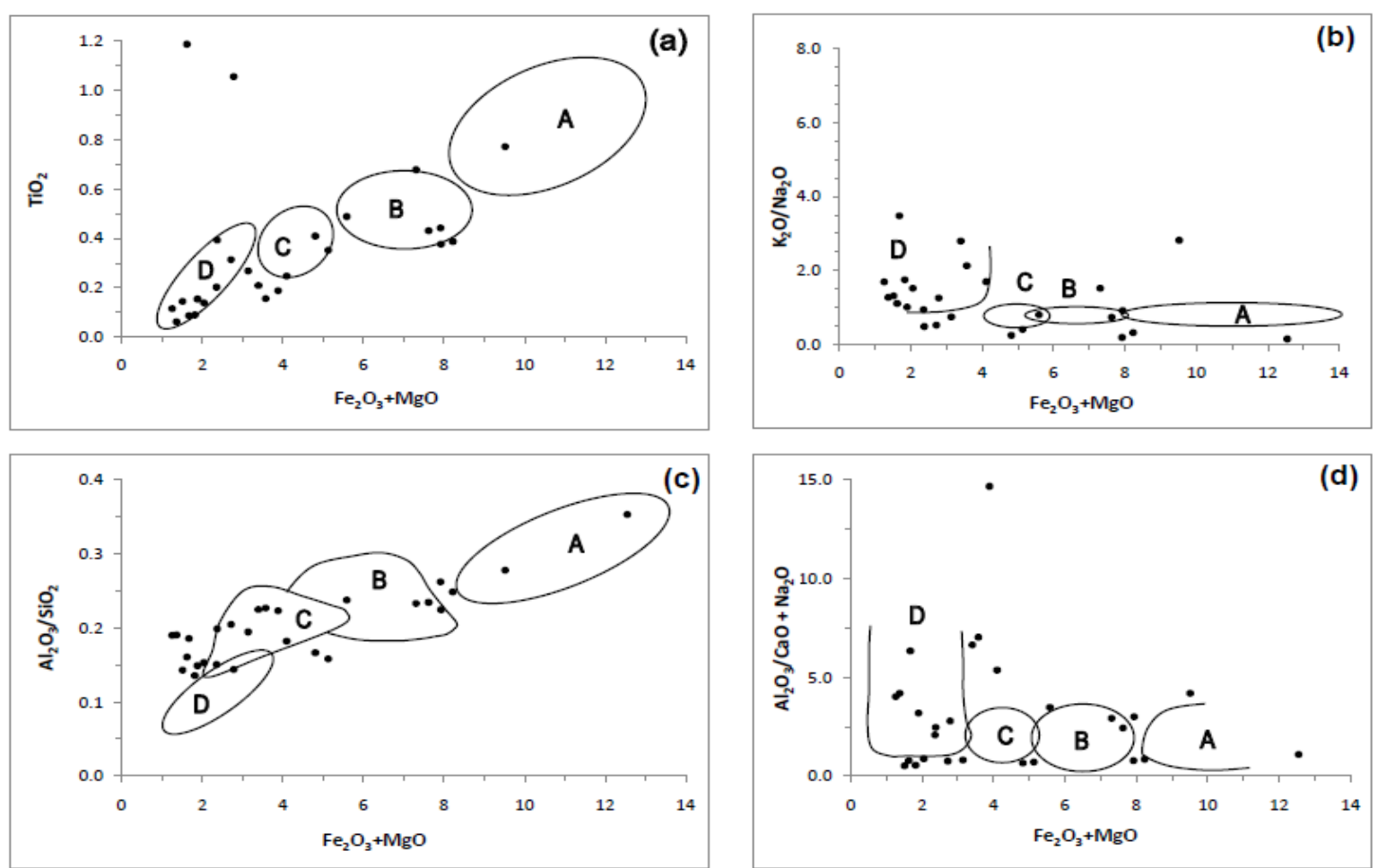

Fig. 9. Plots of (a) $\mathrm{TiO}_{2}$, (b) $\mathrm{K}_{2} \mathrm{O} / \mathrm{Na}_{2} \mathrm{O}$, (c) $\mathrm{Al}_{2} \mathrm{O}_{3} / \mathrm{SiO}_{2}$, and (d) $\mathrm{Al}_{2} \mathrm{O}_{3} /\left(\mathrm{CaO}+\mathrm{Na}_{2} \mathrm{O}\right.$ ) versus $\mathrm{Fe}_{2} \mathrm{O}_{3}+\mathrm{MgO}$ of Bhatia (1983). Tectonic fields are A: Oceanic Island Arc, B; Continental Island Arc, C; Active Continental Margin, and D; Passive Margin.
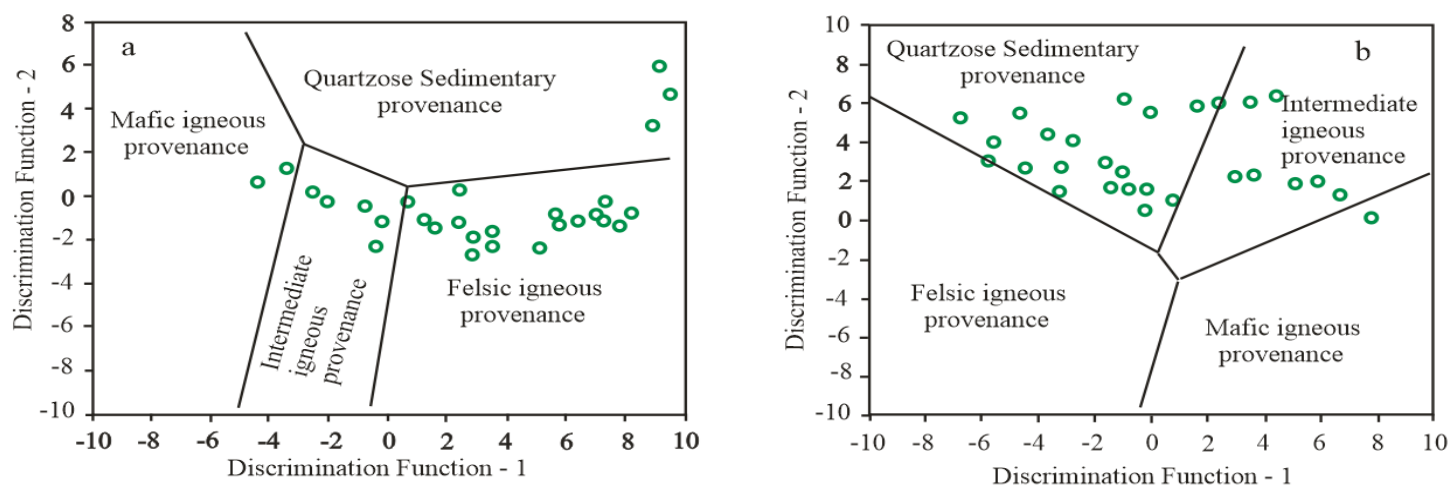

Fig. 10. Discriminant function diagrams of Roser \& Korsch (1988) for Ispikan Conglomerate.

For a), the Discriminant Function-1 is $-1.773 \mathrm{TiO} 2+0.607 \mathrm{Al}_{2} \mathrm{O}_{3}+$

$0.76 \mathrm{Fe}_{2} \mathrm{O}_{3 \text { (Total) }} 1.5 \mathrm{MgO}+0.616 \mathrm{CaO}+0.509 \mathrm{Na}_{2} \mathrm{O}-1.224 \mathrm{~K}_{2} \mathrm{O}-9.09$ and Discriminant Function- 2 is $0.445 \mathrm{TiO}_{2}+0.07 \mathrm{Al}_{2} \mathrm{O}_{3}-0.25 \mathrm{Fe}_{2} \mathrm{O}_{3 \text { (Total) }}-1.142 \mathrm{MgO}+0.438 \mathrm{CaO}+1.475 \mathrm{Na}_{2} \mathrm{O}+1.426 \mathrm{~K}_{2} \mathrm{O}-6.861$.

For b), the Discriminant Function- 1 is $30.638 \mathrm{TiO}_{2} / \mathrm{Al}_{2} \mathrm{O}_{3}-12.541 \mathrm{Fe}_{2} \mathrm{O}_{3 \text { (Total) }} / \mathrm{Al}_{2} \mathrm{O}_{3}+$ $7.329 \mathrm{MgO} / \mathrm{Al}_{2} \mathrm{O}_{3}+12.031 \mathrm{Na}_{2} \mathrm{O} / \mathrm{Al}_{2} \mathrm{O}_{3}+35.402 \mathrm{~K}_{2} \mathrm{O} / \mathrm{Al}_{2} \mathrm{O}_{3}-6.382$, and Discriminant Function-2 is $56.5 \mathrm{TiO}_{2} / \mathrm{Al}_{2} \mathrm{O}_{3}-10.879 \mathrm{Fe}_{2} \mathrm{O}_{3 \text { (Total) }} / \mathrm{Al}_{2} \mathrm{O}_{3}+30.875 \mathrm{MgO} / \mathrm{Al}_{2} \mathrm{O}_{3}-$ $5.404 \mathrm{Na}_{2} \mathrm{O} / \mathrm{Al}_{2} \mathrm{O}_{3}+11.112 \mathrm{~K}_{2} \mathrm{O} / \mathrm{Al}_{2} \mathrm{O}_{3}-3.89$ 

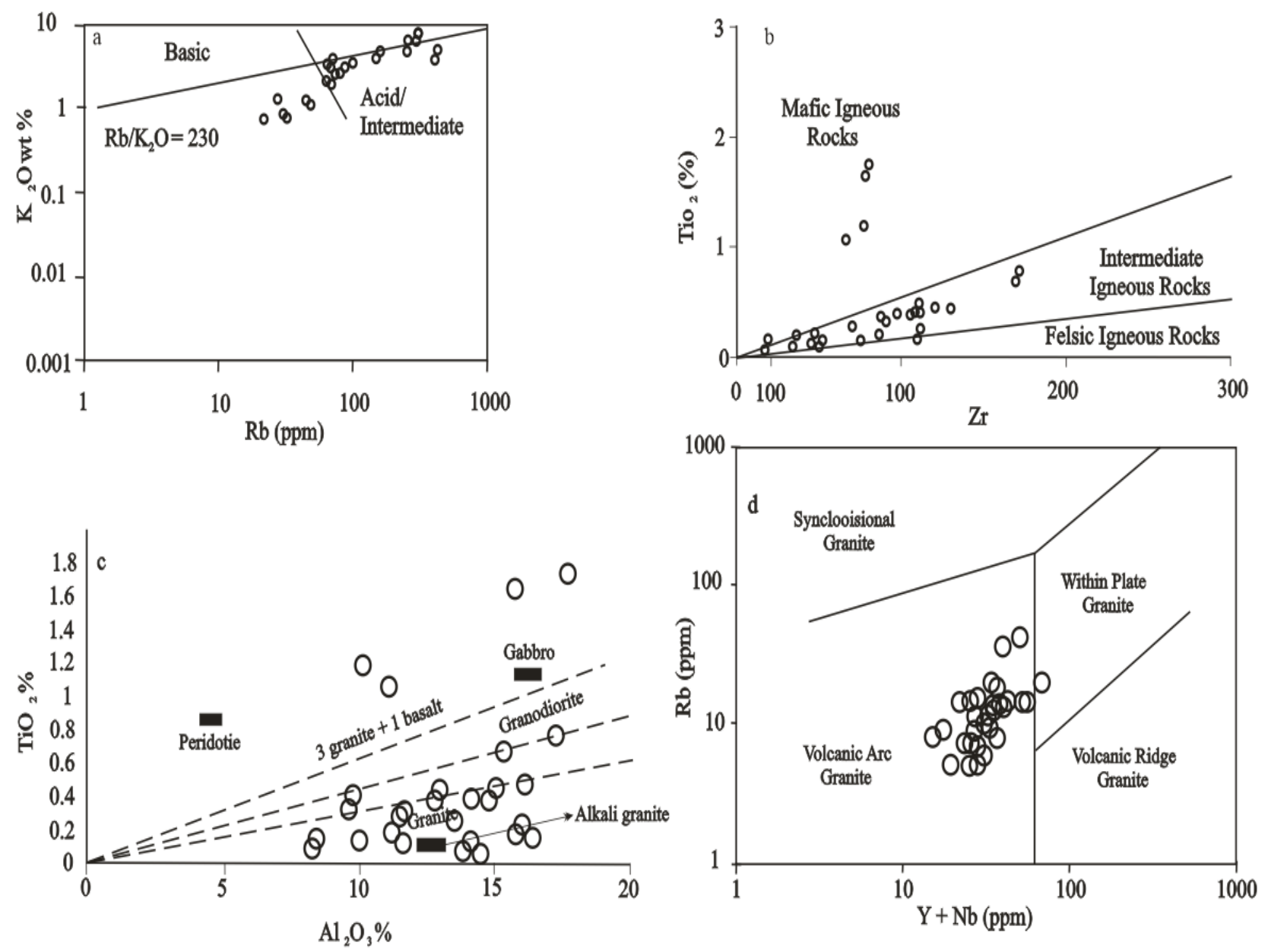

Fig. 11. Scatter plot between

a) $\mathrm{K}_{2} \mathrm{O}$ and $\mathrm{Rb}$ contents (Floyd et al., 1991),

b) $\mathrm{TiO}_{2}-\mathrm{Zr}$, c) $\mathrm{TiO}_{2}-\mathrm{Al}_{2} \mathrm{O}_{3}$ and d) $\mathrm{Rb}-\mathrm{Y}+\mathrm{Nb}$ (Pearce et al., 1984)

of the Ispikan Conglomerate showing derivation of detritus from an acid, intermediate, mafic rocks and volcanic arc granite. 


\begin{tabular}{|c|c|c|c|c|c|}
\hline \multicolumn{2}{|c|}{ Age } & \multicolumn{3}{|c|}{ Litho-stratigraphic Units } & Activities \\
\hline \multicolumn{2}{|c|}{ Holocene } & \multicolumn{3}{|c|}{ Alluvium } & \\
\hline \multirow{2}{*}{\multicolumn{2}{|c|}{ Pleistocene }} & \multicolumn{3}{|c|}{ Jiwani Fm } & Marine shoreline deposits of Ormara and \\
\hline & & \multicolumn{3}{|c|}{ Gwadar Fm } & \\
\hline \multirow{3}{*}{ Pliocene } & Late & \multicolumn{3}{|c|}{ Unconformity } & \\
\hline & \multirow[t]{2}{*}{ Early } & \multirow{4}{*}{ 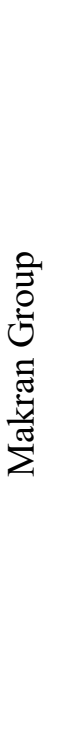 } & \multirow{4}{*}{$\begin{array}{c}\text { Hinglaj Fm./ } \\
\text { Talar } \\
\text { Formation }\end{array}$} & Chatti Mbr & $\begin{array}{l}\text { Neritic, massive, and monotonous sequences of } \\
\text { calcareous mudstones (of Chatti Mudstone) } \\
\text { were deposited. The trench may have shifted } \\
\text { more towards south causing the emergence of } \\
\text { deep marine turbidites on to the land. }\end{array}$ \\
\hline & & & & \multirow{2}{*}{$\begin{array}{c}\text { Hinglaj/ Talar } \\
\text { Mbr }\end{array}$} & $\begin{array}{l}\text { Deposition of Talar-Hinglaj sediments } \\
\text { continued in shallow water conditions. }\end{array}$ \\
\hline Miocene & Middle & & & & \multirow{2}{*}{$\begin{array}{l}\text { The accretionary forearc basin rapidly } \\
\text { prograded from deep marine to shallow marine } \\
\text { or to even coastal environment resulted in the } \\
\text { accumulation of Parkini Mudstone in deeper } \\
\text { water and the Talar Sandstone in shallow water } \\
\text { conditions. }\end{array}$} \\
\hline \multirow{4}{*}{ Oligocene } & \multirow{3}{*}{ Late } & & & Parkini Mbr & \\
\hline & & \multirow{3}{*}{\multicolumn{2}{|c|}{$\begin{array}{l}\text { Khojak } \\
\text { Formation }\end{array}$}} & Shigalu Mbr & $\begin{array}{l}\text { Turbidites of Hoshab Formation, Siahan Shahe } \\
\text { and Panjgoor Formation (Khojak Formation) }\end{array}$ \\
\hline & & & & \multirow{2}{*}{$\begin{array}{c}\text { Murgha } \\
\text { Faqirzai Mbr }\end{array}$} & were deposited in deep marine basin \\
\hline & Early & & & & \\
\hline \multirow{3}{*}{ Eocene } & Late & \multirow{2}{*}{\multicolumn{2}{|c|}{ Wakai Lst }} & Ispikan Congl & $\begin{array}{l}\text { As a result of submarine gravity flow the Ispikan } \\
\text { Conglomerate was slumped down from shallow } \\
\text { water conditions and settled on top of deeper } \\
\text { facies (Marl) of Wakai Limestone. }\end{array}$ \\
\hline & \multirow[t]{2}{*}{ Early } & & & & $\begin{array}{l}\text { Wakai Limestone was deposited during Middle } \\
\text { to Late Eocene in shallow water conditions. It } \\
\text { was a narrow carbonate shelf along the coast of } \\
\text { Makran that submerged later with the influx of } \\
\text { massive turbidites. }\end{array}$ \\
\hline & & \multicolumn{4}{|r|}{ Base not exposed } \\
\hline
\end{tabular}

Fig. 12. Revised stratigraphy of the Makran and new stratigraphic position of the Ispikan Conglomerate. 
Concentrations and inter-element ratios of $\mathrm{Nb}, \mathrm{Zr}, \mathrm{Y}, \mathrm{Rb}, \mathrm{Sr}, \mathrm{Th}, \mathrm{Ba}, \mathrm{Cr}$ and $\mathrm{Ni}$ have also been used to determine the tectonic setting of siliciclastic rocks (Bhatia, 1985). The Ni and Cr contents, and $\mathrm{Zr} / \mathrm{Nb}$ values of Ispikan detritus suggest having been originated from an active continental margin, while the $\mathrm{Th}$ content, $\mathrm{Rb} / \mathrm{Sr}$ values and $\mathrm{Ba} / \mathrm{Sr}$ values closely resemble that of an oceanic island arc. $\mathrm{The} \mathrm{Nb}, \mathrm{Zr} / \mathrm{Th}$ and $\mathrm{Nb} / \mathrm{Y}$ values fall relatively closer to a continental island arc setting.

The $\mathrm{K} / \mathrm{Rb}$ diagram is also used to discriminate sediments derived from an acid and intermediate compositions from those of basic igneous rocks (Floyd et al., 1991). The relatively high $\mathrm{K} / \mathrm{Rb}$ ratio of the sandstones of Ispikan indicates acidic and intermediate igneous provenance (Figure 11a). Similarly, Pearce et al., 1984) used multiple diagrams to identify the parent rock. In such scatter plots, some of the data points are plotted within the acidic region, but most of them are plotted outside, but close to, the specified field of acidic rocks (Figures 11b-c). Similarly, the relationship between $\mathrm{Rb}$ and $\mathrm{Y}+\mathrm{Nb}$ reveals the granitic origin of Ispikan detritus (Figure 11d)

\subsection{Depositional Environment}

Massive debris flows are common in the passive and active continental margins (Canals et al., 2004; Haflidason et al., 2004; Medialdea et al., 2004; Burg et al., 2008, Burg 2018). Active continental margins are more susceptible to have such large-scale catastrophic events producing large-sized Olistostrome. The sedimentary mèlange of Makran Accretionary Prism is a suitable environment for such mega-scale mass flows (Burg et al., 2008). The olistostromes are also common in the flysch rocks and have been reported by many workers (e.g., DiMitrijevic \& DiMitrijevic, 1973; Heubeck, 1992; Delteil et al., 2006, Burg et al., 2008; Cieskowski et al., 2009; 2012). Recently Ruh et al. (2018) have also identified major olistostromes in the Iranian part of the Makran Accretionary Prism creating mini basins for the deposition of thick piles of fine clastic.

Since Miocene, the MAP is going through the Convergence of the northern edge of the Arabian plate into the Eurasian plate, abundant sediment supply, underplating of trench fill sediments causing uplifting and normal faulting, and intensive seismic activities (Platt et al., 1985; Byrne et al., 1992). The combined effects of all these processes are crustal shortening, an extraordinary load of accumulated sediments, tilted and faulted terraces, seaward growth of the accretionary wedge and high magnitude earthquakes. All these effects are sufficient to trigger massive debris flows. Burg et al. (2008) have reported a giant catastrophic the mud-and-debris flow in the Iranian part of the Makran during Miocene (11.8 to 5.8Ma) that produced a huge olistostrome within the Makran turbidites. We concur with this interpretation and postulate that the "Ispikan Debris Flow" may have been a similar event of the Late Eocene or early Oligocene. It may have been a different event in time and space, but this study provides evidence that the Ispikan Conglomerate has a submarine debris flow origin. The petrographic characteristics and the geochemical signatures indicate a proximal source which is only possible with a "debris flow" explanation. 


\subsection{Tectonic History}

The pebbly sandstone and the cobbles and boulders of the Ispikan Conglomerate indicate a relatively short distance of travel. The most plausible nearby source to provide such detritus is the Raskoh Arc which is now located to the north of the Ispikan outcrop. Therefore, the site of deposition was somewhere in the fore-arc basin south of the Raskoh Arc that received volcaniclastic sediments derived from the arc. This interpretation also suggests that from Cretaceous to early Eocene the Raskoh Arc was active and supplied detritus to the nearby forearc basin. Wakai Limestone indicates a shallow and calm marine environment during the early to middle Eocene which changed abruptly in the late Eocene followed by the emplacement of Ispikan detritus. Ispikan Conglomerate represents a short but fast flux of sediments of igneous origin that incorporated sedimentary clasts and fossils during its course of transportation.

The tectonic history of the Chaghi-Raskoh arcs and the surrounding areas has been described by Arthurton \& Farah (1982) whereas the geological history of the Makran area has been discussed in detail by Bender (1995). A more detailed evolutionary history of MAP and associated fault systems have been described by Burg (2018) \& Shah et al. (2021). On a larger regional scale, the tectonic evolution of the Zagros Mountains and associated subduction-accretion processes are comparable with MAP (Aziz \& Sadiq, 2020). By combining the previous studies with the present study, a revised stratigraphic history has emerged and is given in (Figure 12.)

\section{Conclusions}

Ispikan Conglomerate of southwest Makran, a small but important lithologic unit of Makran Accretionary Prism. Its origin, age and the stratigraphic position remained uncertain in the past. Based on this study, it is concluded that sedimentological features, petrography and detrital modes indicate that the provenance of Ispikan detritus was located to the north of the depositional site at a relatively short distance. The source area of Ispikan Conglomerate was composed of plutonic and volcanic rocks with minor metamorphic rocks. The geochemistry of the Ispikan sediments indicates their derivation from an acid and intermediate igneous of the subduction-related volcanic arc.

Ispikan Conglomerate was formed in the late Eocene by a localized submarine debris flow triggered by slope failure, fast sedimentation rate and increased seismic activity. After the placement of Ispikan Conglomerate on the deeper marl facies of Eocene Wakai Limestone, the tectonic environment of the basin was changed. The basin started subsiding and provided space for the accumulation of a thick volume of turbidites in the forearc basin. 


\section{ACKNOWLEDGEMENTS}

This work is the outcome of M.Phil. Thesis research (Khalil ur Rehman) was carried out at the University of Balochistan, Quetta (Pakistan). The financial and logistic support provided by the Centre of Excellence in Mineralogy, University of Balochistan is acknowledged with thanks. Higher Education Commission (Pakistan) is also acknowledged for providing a travel grant to the first author for presenting the paper at $125^{\text {th }}$ Annual Meeting of the GSA at Denver.

\section{References}

Arthurton, R.S., Farah, A. \& Ahmed, W. (1982). The late Cretaceous - Cenozoic history of westran Balochistan, Pakistan, the norhtern margin of the Makran Subduction Complex. - In: Leggett, J.K. (eds): Trench - Forearc Geology. Geological Society London, Special Publications, 10: $373-385$.

Aziz, N.R.H. \& Sadiq, D.M. (2020). U-Pb Zircon dating of upper cretaceous siliciclastic rocks from the Tanjero Flysch, NE Iraq: New constraints on their provenance and tectonic evolution. Kuwait Journal of Science, 47(4): 106-117.

Bender, F.K. (1995). Geological framework. In F.K. Bender and H.A. Raza, (eds) Geology of Pakistan. Publication in Berlin, 11-61.

Bender, F.K. \& Raza, H.A. (1995). Geology of Pakistan. GB Berlin-Stuttgart, Germany, 414 p.

Bhatia, M.R. (1983). Plate tectonics and geochemical compositions of sandstones. Journal of Geology, 91: 611-627.

Bhatia, M.R. (1985). Rare earth element geochemistry of Australian Paleozoic graywackes and mudrocks: provenance and tectonic control. Sedimentary Geology, 45: 97-113.

Burg, Jean-Pierre (2019). Late Miocene Olistostrome in the Makran Accretionary Wedge (Baluchistan, SE Iran): A Short Review in Ogata, K., Festa, A., Pini, G. A. (Eds.) Submarine Landslides: Subaqueous Mass Transport Deposits from Outcrops to Seismic Profiles American Geophysical Union. 45-55.

Burg, Jean-Pierre (2018). Geology of the onshore Makran Accretionary wedge: Synthesis and tectonic interpretation. Earth-Science Reviews, 185: 1210-1231

Burg, J.P., Bernoulli, D. Smit, J., Dolati, A. \& Bahroudi, A. (2008). A giant catastrophic mudand-debris flow in the Miocene Makran. Terra Nova, 20: 188-193.

Byrne, D.E., Sykes, L.R. \& Davis, D.M. (1992). Great thrust earthquakes and aseismic slip along the plate boundary of the Makran Subduction Zone. Journal of Geophysical. Research, 97 (B1): $449-478$. 
Canals, M., Lastras, G., Urgeles, R., Casamor, J.L., Mienert, J., Cattaneo, A., De batist, M., Haflidason, H., Imbo, Y., Laberg, J.S., Locat, J., Long, D., Longva, O., Nasson, D.G., Sultan, N., Trincardi, F. \& Bryn, P. (2004). Slope failure dynamics and impacts from seafloor and shallow sub-seafloor geophysical data: case studies from the COSTA project. Marine and Geology, 213: 9-72

Cieszkowski, M., Golonka, J., Ślączka, A. \& Waśkowska, A. (2012). Role of the olistostromes and olistoliths in tectonostratigraphic evolution of the Silesian Basin in the Outer West Carpathians. Tectonophysics, 568: 248-265.

Cieszkowski, M., Golonka, J., Krobicki, M., Ślączka, A., Oszczypko, N., Waśkowska, A. \& Wendorff, M. (2009). The Northern Carpathian plate tectonic evolutionary stages and origin of olistolithes and olistostromes. Geodinamica Acta, 22(1-3): 101-126.

Crook, K.A.W. (1974). Lithogenesis and geotectonics: the significance of compositional variations in flysch arenites (graywackes), In Dott, R.H. Jr., and R.H. Shaver, (eds). Modern and ancient geosynclinal sedimentation. Society of Economic. Paleontologists and Mineralogists Special Publication, 19: 304-310.

Delteil, J., Mercier De Lepinay, B., Morgans, H.E.G. \& Field, B.D. (2006). Olistostromes marking tectonic events, East Coast, New Zealand. New Zealand Journal of Geology and Geophysics, 49: 571-531.

DiMitrijevic, M.D. \& DiMitrijevic M.N. (1973). Olistostrome Melange in the Yogoslavian Dinarides and Late Masozoic plate tectonics, Journal of Geology, 81(3): 329-340.

Dickinson, W.R. (1985). Interpreting provenance relations from detrital modes modes of sandstones. In Zuffa, G. G., ed. Provenance of Arenites: D. Reidel, Dordrecht, 333-361.

Dickinson, W.R. (1988). Provenance of Sediment Dispersal in relation to Paleotectonics and Paleography of Sedimentary Basins. In Kleinspehn, K. L., and Paola, C. (ed) "New Perspectives in Basin Analysis," Springer Verlog, 3-25.

Dickinson, W.R. \& Suczek, C.A. (1979). Plate tectonics and sandstone composition: American Association of Petroleum Geologists Bulletin, 63(12): 2164-82.

Farhoudi, G. \& Karig, D.E. (1977). Makran of Iran and Pakistan as an active arc system: Geology, 5: 664-668.

Farooqui, M.A. \& Rehman, K.U. (2013). Petrography, geochemistry and depositional model of Ispikan Conglomerate, Makran Accretionary Prism, Southwest Pakistan [abs.]. 125 ${ }^{\text {th }}$ Anniversary Meeting of the Geological Society of America Abstract with Program; Paper No. 92-7: 45/7.

Floyd, P.A., Shail, R., Leveridge, B.E. \& Frank. W. (1991). Geochemistry and provenance of Rhenohercynian Synorgenic sandstone: implications for tectonic environment discrimination; In Morton, A. C., Todd, S. P and Haughton, P. D. (eds) Developments in Sedimentary Provenance Studies. Geological Society of London Special Publication, 57: 173-198. 
Haflidason, H., Sejrup, H.P., Nygard, A., Mienert, J., Bryn, P., Lien, R., Forsberg, C.F., Berg, K. \& Masson, D. (2004). The Storegga Slide: architecture, geometry and slide development. Marine Geology, 213: 201-234.

Haughton, P.D.W. (1988). A cryptic Caledonian flysch terrane in Scotland. Journal of the Geological Society of America Bulletin, 95: 1261- 1267.

Heuback, C. (1992). Sedimentology of Large Olistoliths, Southern Cordillera Central, Hispaniola; Journal of Sedimentary Petrology, 62(3): 474-482.

Hiscott, R.N. (1984). Ophiolitic source rock for Taconic-age flysch; trace elements evidence. Geological Society of America Bulletin, 95: 1261-1267.

HSC (Hunting Survey Corporation) (1960). Reconnaissance geology of part of West Pakistan. A Colombo Plan Co-operative Project. Government of Canada, Toronto, 550p.

Huang, Y., Guangqing, Y. \& Fengde Z. (2018). Provenance analysis for submarine fan sandstones of Huangliu Formation, Dongfang 13 gas field in Yinggehai Basin, South China Sea: Kuwait Journal of Science, 45(3): 72-92.

Kassi, A.M., Khan, A.S. \& Kasi, A.K. (2007). Newly proposed Cretaceous-Paleocene lithostratigraphy of the Ispikan-Wakai area, southwestern Makran, Pakistan. Journal of Himalayan Earth Sciences, 40: 25-31.

Malkani, S. M. \& Mahmood, Z. (2017). Stratigraphy of Pakistan. Memoir 24, Geological Survey of Pakistan, 137p.

McCall, G.J.H. \& Kidd, R.G.W. (1982). The Makran, Southeastern Iran; the anatomy of a convergent plate margin active from cretaceous to present. - In: Leggett, J.K (eds): Trench Forearc Geology, Sediment, and tectonics on Modern and a next active plate margin. Geological. Society of London Special Publication, 10: 387-397.

McCann, T. (1991). Petrological and geochemical determination of provenance in a beckare basin, Halmahere, eastern Indonesia in Morton, A.C. et.al. (eds) Developments in Sedimentary Provenance Studies. Geological Society of London Special Publication, 57: 215-230.

Medialdea, T., Vegas, R., Somoza, L., Va'zquez, J.T., Maldonado, A., Dı'az-delRı'o, V., Maestro, A., Co'rdoba, D. \& Ferna'ndez-Puga, M.C. (2004). Structure and evolution of the "Olistostrome" complex of the Gibraltar Arc in the Gulf of Ca' diz (eastern Central Atlantic): evidence from two long seismic cross-sections. Marine Geology, 209: 173-198.

Pearce, J.A., Harris, N.B.W. \& Tindle, A.G. (1984). Trace element discrimination diagrams for the tectonic interpretation of granitic rocks; Journal of Petrology, 25: 956-983.

Penney, C., Tavakoli, F., Saadat, A., Nankali, H.R., Sedighi, M., Khorrami, F., Sobouti, F., Rafi, F., Copley, A., Jackson, J. \& Priestley, K., (2017). Megathrust and accretionary wedge 
properties and behavior in the Makran subduction zone. Geophysical Journal International, 209: 1800-1830.

Platt, J.P. \& Leggett, J.K. (1986). Stratal Extension in thrust footwalls, Makran Acrretionary Prism; implications for thrust tectonics. American Association of Petroleum Geologists Bulletin, 70(2): 191-203.

Platt, J.P., Leggett, J.K., Young, J., Raza, H. \& Alam, S. (1985). Large-scale sediment underplating in the Makran Accretionary prism. Geology, 13(7), 507-511.

Raza, H.A. \& Alam, S. (1983). Pakistan's Makran region merits oil hunt. Oil and Gas Journal, 81: $170-174$.

Rehman, Khalil-Ur, (2002). Provenance and Petrology of Paleocene (?) Ispikan Conglomerate, Southwest Makran, and its Implications on the Tectonic Evolution of Makran Region, Pakistan. M.Phil. thesis (unpubl.), Centre of Excellence in Mineralogy, University of Balochistan, Quetta. 206 p.

Roser, B.P. \& Korsch, R.J. (1986). Determination of tectonic setting of sandstone - mudstone suites using $\mathrm{SiO}_{2}$ content and $\mathrm{K}_{2} \mathrm{O} / \mathrm{Na}_{2} \mathrm{O}$ ratio. Journal of Geology, 94: 635-50.

Ruh, Jonas B., Vergés, J. \& Burg, Jean-Pierre (2018). Shale-related minibasins atop a massive olistostrome in an active accretionary wedge setting: Two-dimensional numerical modeling applied to the Iranian Makran. Geology, 46(9): 791-794.

Shah, S.T., Ozacar, A.A. \& Gülerce, Z. (2021). Fault-based probabilistic seismic hazard assessment of the eastern Makran subduction and the Chaman transform fault, Pakistan: Emphasis on the source characterization of megathrust; Journal of Asian Earth Sciences, 205: 1-19.

Smith, G.L., McNeill, L.C., Wang, K., He, J. \& Henstock, T.J. (2013). Thermal structure and megathrust seismogenic potential of the Makran subduction zone. Geophysical Research Letters, 40: $1528-1533$.

Wrafter, J.P. \& Graham, J.R. (1989). Ophiolitic detritus in the Ordovician sediments of South Mayo, Ireland. Journal of the Geological Society of London, 146: 213-215.

Submitted: 01/09/2020

Revised: $12 / 03 / 2021$

Accepted: 20/03/2021

DOI: $10.48129 / \mathrm{kjs} . v 49 \mathrm{i} 1.10486$ 